\title{
Recoil detection of the lightest neutralino in MSSM singlet extensions
}

\author{
Vernon Barger, ${ }^{1}$ Paul Langacker, ${ }^{2}$ Ian Lewis, ${ }^{1}$ Mat McCaskey, ${ }^{1}$ Gabe Shaughnessy, ${ }^{1}$ and Brian Yencho ${ }^{1}$ \\ ${ }^{1}$ Department of Physics, University of Wisconsin, Madison, Wisconsin 53706, USA \\ ${ }^{2}$ School of Natural Sciences, Institute for Advanced Study, Einstein Drive, Princeton, New Jersey 08540, USA
}

(Received 23 February 2007; published 1 June 2007)

\begin{abstract}
We investigate the correlated predictions of singlet extended MSSM models for direct detection and the cosmological relic density of the lightest neutralino. To illustrate the general effects of the singlet, we take heavy sleptons and squarks. We apply CERN LEP, $(g-2)_{\mu}$, and perturbativity constraints. We find that the WMAP upper bound on the cold dark matter density limits much of the parameter space to regions where the lightest neutralino can be discovered in recoil experiments. The results for the next-to-minimal supersymmetric standard model and $U(1)^{\prime}$-extended minimal supersymmetric standard model are typically similar to the MSSM since their light neutralinos have similar compositions and masses. In the nearly minimal supersymmetric standard model the neutralino is often very light and its recoil detection is within the reach of the CDMS II experiment. In general, most points in the parameter spaces of the singlet models we consider are accessible to the WARP experiment.
\end{abstract}

DOI: 10.1103/PhysRevD.75.115002

PACS numbers: 12.60.Jv, 95.35.+d

\section{INTRODUCTION}

The evidence for cold, nonbaryonic dark matter is one of the strongest cases for physics beyond the standard model (SM). The most plausible candidates are axions and weakly interacting massive particles (WIMPs). Many models have been proposed that provide a WIMP candidate for dark matter (DM). These models include supersymmetry [1-3], extra dimensions [4,5], little Higgs [6-8], and twin Higgs models $[9,10]$. It is anticipated that the CERN LHC and ILC may directly produce the DM particle, providing crucial information on its existence, origin, and properties [11-18]. Recoils of nuclei from the scattering of WIMPs can also provide direct evidence for this DM candidate $[19,20]$. Alternative avenues exist to discover the DM particle indirectly. These include observation of gamma ray lines in the galactic halo peaked at the neutralino mass [21,22], observation of high energy neutrinos from WIMP annihilation in the sun [23-25], and antiparticle detection [26-29].

The minimal supersymmetric standard model (MSSM) with conserved $R$ parity has a viable dark matter candidate, the lightest neutralino, that can naturally explain the abundance of cold dark matter (CDM) in the Universe. Other motivations for the MSSM include solutions to the gauge hierarchy problem, the quadratic divergence in the Higgs boson mass, and gauge coupling unification [30-32]. The MSSM Lagrangian contains a Higgsino mixing parameter, $\mu$, the only massive parameter of the model that conserves supersymmetry. The $\mu$ parameter must be at the electroweak (EW) or $\mathrm{TeV}$ scale to explain electroweak symmetry breaking. This is the so-called hierarchy problem of supersymmetry because $a$ priori the value of $\mu$ is arbitrary [33]. The problem may be resolved by promoting the $\mu$ parameter to a dynamical field whose vacuum expectation value $\langle S\rangle$ and coupling $\lambda$ determine the effective $\mu$ parameter,

$$
\mu_{\text {eff }}=\lambda\langle S\rangle \text {. }
$$

Singlet extended models (xMSSM) have significant consequences for the Higgs and neutralino sectors [34-47].

In this paper we focus on some of the proposed singlet extensions of the MSSM: (i) the next-to-minimal supersymmetric SM (NMSSM) [48-50] with a trilinear singlet term in the superpotential, (ii) the nearly minimal supersymmetric SM (nMSSM) [51-54] with a tadpole term in the superpotential, and (iii) the $U(1)^{\prime}$-extended MSSM (UMSSM) [55-57] with an extra $Z^{\prime}$ gauge boson, as detailed in Table I along with the respective symmetries and numbers of neutralino, chargino, and Higgs states. A secluded $U(1)^{\prime}$-extended MSSM (sMSSM) [35,58] with three singlets in addition to the standard UMSSM Higgs singlet is equivalent to the nMSSM in the limit that the additional singlet vacuum expectation values (vevs) are large and the trilinear singlet coupling, $\lambda_{s}$, is small [37]. Thus, we collectively refer to the nMSSM and SMSSM as the $\mathrm{n} / \mathrm{sMSSM}$.

Other singlet extensions are possible, and often are derived from string constructions. The UMSSM is an example of such string constructions [56,59-62]. For recent reviews and articles, see, e.g., Refs. [59-66]. For a review of supersymmetric singlet models, see Ref. [34]. The SM may also be extended to include a singlet. Depending on the parity of the singlet, it may mix with the Higgs boson $[67,68]$ or provide a viable dark matter candidate [69-71].

The extended MSSM models (xMSSM) have extra neutralino and Higgs states, that affect the spectra and composition of the particle states [36,38], and significantly influence the parameter space for the observation via direct or indirect detection of neutralino dark matter. For example, the processes involved in indirect detection have been shown to be radiatively enhanced by the extended Higgs sector in the NMSSM [72]. The direct detection prospects have been recently investigated in the NMSSM $[73,74]$. The relic density of the lightest neutralino can also 
TABLE I. Symmetries associated with each model and their respective terms in the superpotential; the number of states in the neutralino and Higgs sectors are also given. All models have two charginos, $\chi_{i}^{ \pm}$, and one charged Higgs boson, $H^{ \pm}$.

\begin{tabular}{c|ccccc}
\hline \hline Model & MSSM & NMSSM & nMSSM & UMSSM & sMSSM \\
\hline Symmetry & $\cdots$ & $\mathbb{Z}_{3}$ & $\mathbb{Z}_{5}^{R}, \mathbb{Z}_{7}^{R}$ & $U(1)^{\prime}$ & $U(1)^{\prime}$ \\
Extra superpotential term & $\cdots$ & $\frac{\kappa}{3} \hat{S}^{3}$ & $t_{F} \hat{S}$ & $\cdots$ & $\lambda_{S} \hat{S}_{1} \hat{S}_{2} \hat{S}_{3}$ \\
& $\cdots$ & $($ cubic) & (tadpole) & $\cdots$ & (trilinear secluded) \\
$\chi_{i}^{0}$ & 4 & 5 & 5 & 6 & 9 \\
$H_{i}^{0}$ & 2 & 3 & 3 & 3 & 6 \\
$A_{i}^{0}$ & 1 & 2 & 2 & 1 & 4 \\
\hline \hline
\end{tabular}

be dependent on the model; the density relative to the closure density is very roughly given by the total annihilation cross section by [75]

$$
\Omega_{\chi_{1}^{0}} h^{2} \simeq \frac{0.1 \mathrm{pb}}{\left\langle\sigma_{\mathrm{ann}} v\right\rangle},
$$

where $v$ is the relative velocity and $\sigma_{\text {ann }}$ includes effects from neutralino coannihilation with particles of similar mass. The nonbaryonic dark matter relic density relative to the critical density, $\Omega_{\mathrm{DM}}$, is determined by the WMAP 3 -year cosmic microwave background data and the spatial distribution of galaxies to be $[75,76]$

$$
\Omega_{\mathrm{DM}} h^{2}=0.111 \pm 0.006
$$

where $h=0.74 \pm 0.03$ is the Hubble constant. However, there could be multiple origins of dark matter, such as neutralinos and axions or nonthermal production, so, strictly speaking, Eq. (4) provides only an upper bound on neutralino dark matter.

In this paper we consider the neutralino and Higgs states of the singlet extended supersymmetric models. The predictions for the neutralino relic density and neutralinonucleon scattering cross section are often correlated. We find that the singlet and singlino admixtures of the Higgs bosons and neutralinos, respectively, can dramatically alter the predicted relic density and the direct detection scattering cross section. We calculate the xMSSM predictions, along with those of the MSSM, ${ }^{1}$ with the parametrization of the Higgs and neutralino sectors given in Ref. [37]. Many studies on the relic density and direct detection of the lightest neutralino have been performed in the constrained MSSM (CMSSM) which imposes scalar mass, gaugino mass, and soft trilinear mass unification at the grand unified theory (GUT) scale, thereby reducing the number of parameters in the model [77-80]. Additional work on the connection between the Higgs sector and

\footnotetext{
${ }^{1}$ Note that the version of the MSSM we adopt is more general than minimal supergravity (mSUGRA) since we set parameters at the $\mathrm{TeV}$ scale.
}

astrophysical constraints in the CMSSM has also been done in the MSSM [14,81]. More general recent analyses of the MSSM can be found in Refs. [16,82].

In Sec. II, we analyze the neutralino annihilation and coannihilations and how the current value of $\Omega_{\mathrm{DM}} h^{2}$ constrains the Higgs and neutralino spectra. In Sec. III we consider neutralino-nucleon scattering and the prospects for direct detection of neutralino dark matter in the xMSSM models. Finally, in Sec. IV, we summarize our conclusions and provide an outlook on the discovery of the lightest neutralino for the models we consider. We reserve the appendixes for discussions of the neutralino mass matrix of the extended models and couplings which are altered or completely new relative to the MSSM. Further, we discuss the constraints from the $(g-2)_{\mu}$ measurement and perturbativity of the couplings.

\section{NEUTRALINO ANNIHILATION AND COANNIHILATION}

The light neutralino states in singlet extended models often have an appreciable singlino component or are dominantly singlino. The singlino admixture has a strong influence on the neutralino annihilation cross section and consequently on the neutralino relic density [38,83]. Coannihilations with supersymmetric particles of similar mass may also significantly affect the relic density.

We calculate the relic abundance of the lightest neutralino in each model using appropriate modifications of the DARKSUSY (DS) code [84]. ${ }^{2}$ We change the Higgs and neutralino couplings in DS to account for the additional interactions of the singlets and singlinos (and $Z^{\prime}$-inos in the UMSSM). We list the couplings in Appendix B. The number of Higgs and neutralino states in DS are increased to account for the additional states in the extended models relative to the MSSM. The Higgs boson width is recalculated for the different spectra and changes in couplings. ${ }^{3}$

\footnotetext{
${ }^{2}$ Other supersymmetric dark matter codes exist, including MICROMEGAS [85] and ISATOOLS [86].

${ }^{3}$ We also include the Higgs decay to off-shell gauge bosons, which can be important for intermediate Higgs masses $\left(120 \mathrm{GeV} \lesssim M_{H_{i}} \lesssim 2 M_{Z}\right)$.
} 
MSSM
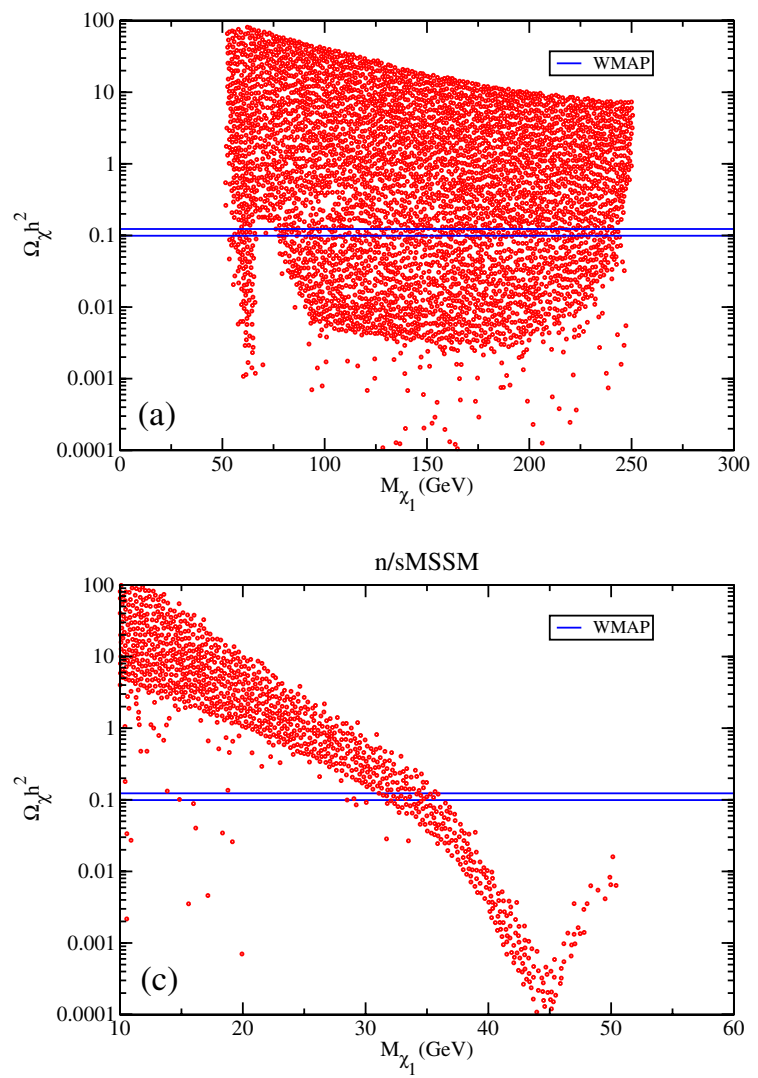

NMSSM
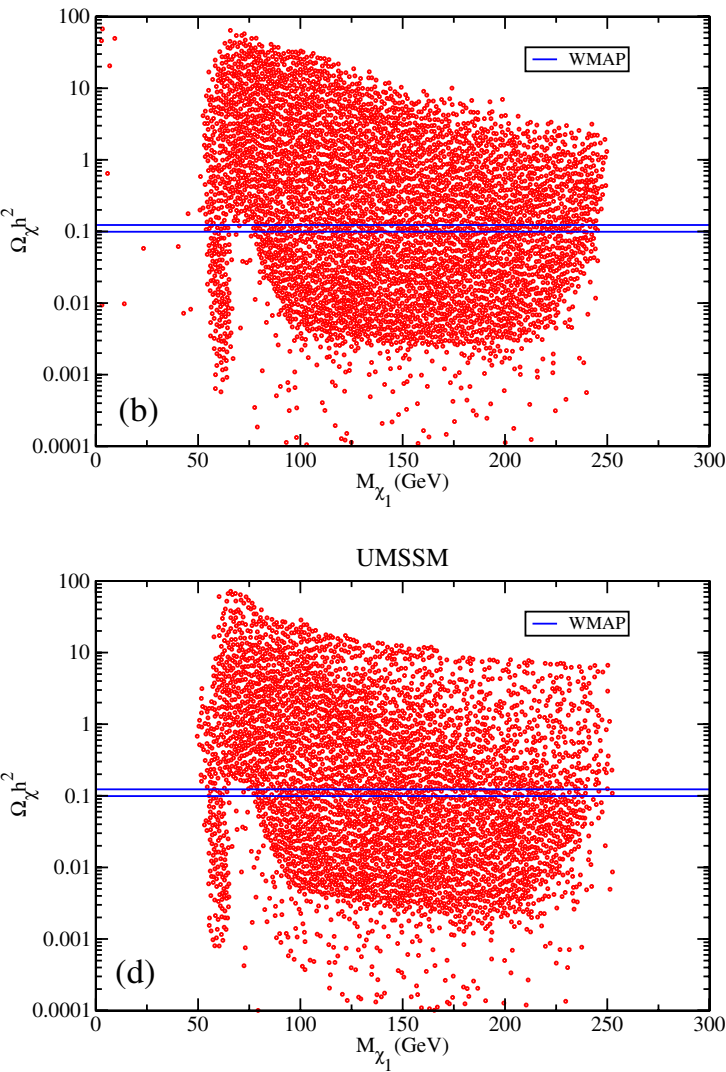

FIG. 1 (color online). Neutralino relic density versus the lightest neutralino mass. The relic density is constrained to be in the region $0.123>\Omega_{\mathrm{DM}} h^{2}>0.099$ provided that the model is solely responsible for the observed dark matter. The efficient annihilations through the Higgs boson pole in the MSSM, NMSSM, and UMSSM are evident at $m_{\chi_{1}^{0}} \sim M_{H_{1}} / 2 \sim 60 \mathrm{GeV}$ and of the $Z$ boson pole at $m_{\chi_{1}^{0}} \sim M_{Z} / 2$ in the $n / s M S S M$.

Our calculation of the thermally averaged annihilation cross section at tree level in our modified version of DS includes all possible initial, final, and exchanged particles of the xMSSM. ${ }^{4}$ For general discussion of the relic density calculation, see Ref. [87].

Because of coannihilations with the extra neutralino states in XMSSM models, the relic density can be different from the MSSM result. While Boltzmann suppression makes contributions from particles with masses $\gtrsim 1.4 m_{\chi_{1}^{0}}$ small, we include coannihilations from particles with masses up to $2.5 m_{\chi_{1}^{0}}$ (Ref. [88] included coannihilations up to $\left.2.1 m_{\chi_{1}^{0}}\right)$.

We fix the slepton and squark masses at a high value $(5 \mathrm{TeV})$ to focus our attention on the effects the singlet has on the relic density. Therefore, these scalar sparticles do not coannihilate with the neutralino, making their effect on

\footnotetext{
${ }^{4}$ Note that we do not include exotic states that can be present in some extended models. For a list of the initial, final, and exchanged states considered by DARKSUSY in the MSSM, see Table 3 in Ref. [84].
}

the relic density negligible. ${ }^{5}$ The $\mathrm{n} / \mathrm{sMSSM}$ supersymmetric spectrum is typically heavy compared to the lightest neutralino, making the contribution to the relic density from coannihilations negligible. In the MSSM, NMSSM, and UMSSM, if the relic density without the effect of coannihilations is at or above the observed value of the relic density, the coannihilation contribution has little to no effect on the relic density [88]. In this region, the neutralino is dominantly $B$-ino, making the coupling, and therefore the coannihilation rate with the $W$-ino-dominated $\chi_{2}^{0}$ and $\chi_{2}^{ \pm}$, small. ${ }^{6}$

The calculated relic density of the neutralino in the various models is shown in Fig. 1 versus the mass of $\chi_{1}^{0}$. The region $0.123>\Omega_{\mathrm{DM}} h^{2}>0.099$ is the $2 \sigma$ observed range [75,76]. Values $\Omega_{\chi_{1}^{0}} h^{2}<0.099$ are allowed in the event that other particles contribute to the relic abundance

\footnotetext{
${ }^{5}$ Of course, additional coannihilation solutions would be possible in all models for lighter squarks and sleptons.

${ }^{6}$ Again, assuming the sleptons are heavy, thereby suppressing the coannihilation process $\chi_{1}^{0} \chi_{i}^{0} \rightarrow f \bar{f}$.
} 


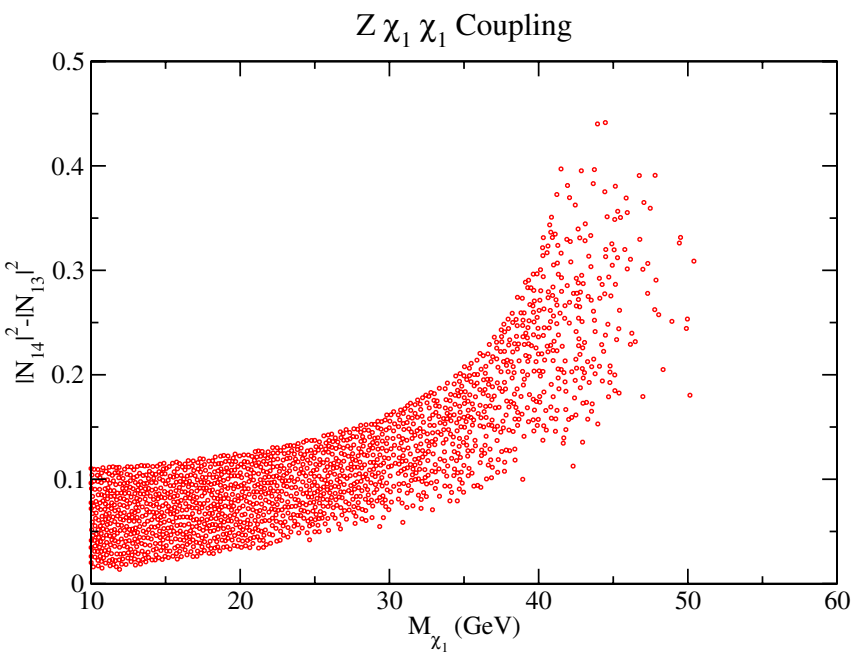

FIG. 2 (color online). The $Z \chi_{1}^{0} \chi_{1}^{0}$ coupling $\left(\propto\left|N_{14}\right|^{2}-\right.$ $\left|N_{13}\right|^{2}$ ) decreases in the $\mathrm{n} / \mathrm{sMSSM}$ as the neutralino mass decreases, assisting in the enhancement of the relic density in the low mass region.

of dark matter, or there is an enhanced nonthermal production mechanism.

The profiles of the relic density versus the lightest neutralino mass are similar for the MSSM, NMSSM, and UMSSM due to their similar low energy neutralino spectra. In the NMSSM and UMSSM, the singlino is typically substantially heavier than the lightest neutralino, $\chi_{1}^{0}$, preventing a large singlino mixture in $\chi_{1}^{0}$. However, the NMSSM allows a lower neutralino mass than in the UMSSM and MSSM, as the $\kappa \rightarrow 0$ limit resembles the $\mathrm{n} /$ sMSSM which has a very light $\chi_{1}^{0} .{ }^{7}$

Overall, there is a broad filled region in Fig. 1 for the MSSM, NMSSM, and UMSSM where the relic density varies depending on the composition and mass of the lightest neutralino. For gaugino dark matter, the annihilation rate is not strong enough to yield the observed dark matter. As the Higgsino content increases, the relic density decreases as the annihilation rate becomes larger. The suppression of the relic density due to efficient annihilation through the $H_{1}$ pole near $m_{\chi_{1}^{0}} \sim 60 \mathrm{GeV}$ is evident. The $H_{2}$ pole may also have a similar effect, as indicated by the sporadic points falling below the filled regions. In this low region, namely $\Omega_{\chi_{1}^{0}} h^{2} \lesssim$ few $\times 10^{-3}$, the NMSSM and UMSSM have more points than the MSSM since there are more Higgs resonances that contribute.

For the n/sMSSM, the lightest neutralino mass is usually below $50 \mathrm{GeV}[53,83]$. Annihilation through the $Z$ boson

\footnotetext{
${ }^{7}$ This corresponds to the PQ limit of the NMSSM. In this limit, both $A_{1}$ and $\chi_{1}^{0}$ may be light, sometimes yielding the observed relic abundance of DM due to efficient annihilation through the $A_{1}$ pole. Constraints on the very light neutralino states can be found when considering limits on the invisible decays of quarkonium states [89].
}

pole dominates the rate near $m_{\chi_{1}^{0}} \approx 45 \mathrm{GeV}$, decreasing the relic abundance below the observed value. Additionally, in the n/sMSSM the relic density is strongly dependent on the mass of the lightest neutralino. As the neutralino mass decreases, the annihilation rate becomes suppressed by the $Z$ propagator, increasing the relic density. Furthermore, in the $\mathrm{n} / \mathrm{sMSSM}$, there is a $Z \chi_{1}^{0} \chi_{1}^{0}$ coupling suppression since the neutralino increasingly becomes singlino at lower $m_{\chi_{1}^{0}}$ as shown in Fig. 2. Therefore, an approximate lower bound on the lightest neutralino mass in the n/sMSSM can be placed at $m_{\chi_{1}^{0}} \geq 30 \mathrm{GeV}$.

Some points in the n/sMSSM exist below the $30 \mathrm{GeV}$ $m_{\chi_{1}^{0}}$ bound where the relic density falls at or below the observed value due to the contribution of additional channels. For example, a light $A_{1}$ resonance enhances the annihilation cross sections. Similarly, the $A$-funnel region in mSUGRA parameter space $[90,91]$ can account for the observed relic density. However, in the present case, the lightest $C P$-odd Higgs is singlet. Excluding annihilation through the $A$ pole, the neutralino mass range compatible with the lower and upper bounds of $\Omega_{\mathrm{DM}}$ is $30 \mathrm{GeV} \lesssim m_{\chi_{1}^{0}} \lesssim 37 \mathrm{GeV}$. ${ }^{8}$ The limit might also be slightly weakened in the full secluded model $[35,58]$ which may include a singlino $\chi_{0}^{0}$ even lighter than the $\chi_{1}^{0}$. Even a tiny $\chi_{0}^{0}-\chi_{1}^{0}$ mixing, irrelevant for collider physics, could allow $\chi_{1}^{0}$ to decay, e.g., to $\chi_{0}^{0} f \bar{f}$, reducing $\Omega_{\chi^{0}} h^{2}$ by $m_{\chi_{0}^{0}} / m_{\chi_{1}^{0}}[92]$.

To further document the effect of the $H_{1}$ pole on the relic density, we plot in Fig. 3 the masses of the lightest Higgs boson and neutralino for various ranges of the relic density. In models with singlet mixing, the lightest Higgs mass can be lower than in the MSSM, filling the major band of $114 \mathrm{GeV}<M_{H_{1}}<135 \mathrm{GeV}$.

In Fig. 3, the MSSM, NMSSM, and UMSSM show bands of allowed points associated with the $H_{1}$ pole near the line $m_{\chi_{1}^{0}} \simeq 2.1 m_{H_{1}}$. The overdense region where $m_{\chi_{1}^{0}} \sim$ $75 \mathrm{GeV}$ is due to inefficient annihilation of gauginolike neutralinos. In the NMSSM, there are points with low allowed $H_{1}$ masses near this band; most points there are due to annihilation through a SM-like $\mathrm{H}_{2}$.

The n/sMSSM shows a weak dependence on the Higgs mass since the most dominant annihilation is through the $Z$ boson. The vertical bands of accepted points are due to the dependence of the relic density on the lightest neutralino mass, as discussed above.

Figure 4 shows the relic density in the plane of $\mu_{\text {eff }}$ and the lightest neutralino mass. Two bands of allowed relic density values are evident in the MSSM, NMSSM, and

\footnotetext{
${ }^{8}$ Since we only consider sfermion masses much heavier than the neutralino, coannihilation with these particles does not contribute to the effective annihilation rate. In general, slepton and squark coannihilation can be important as we parametrize the MSSM more generally than mSUGRA.
} 

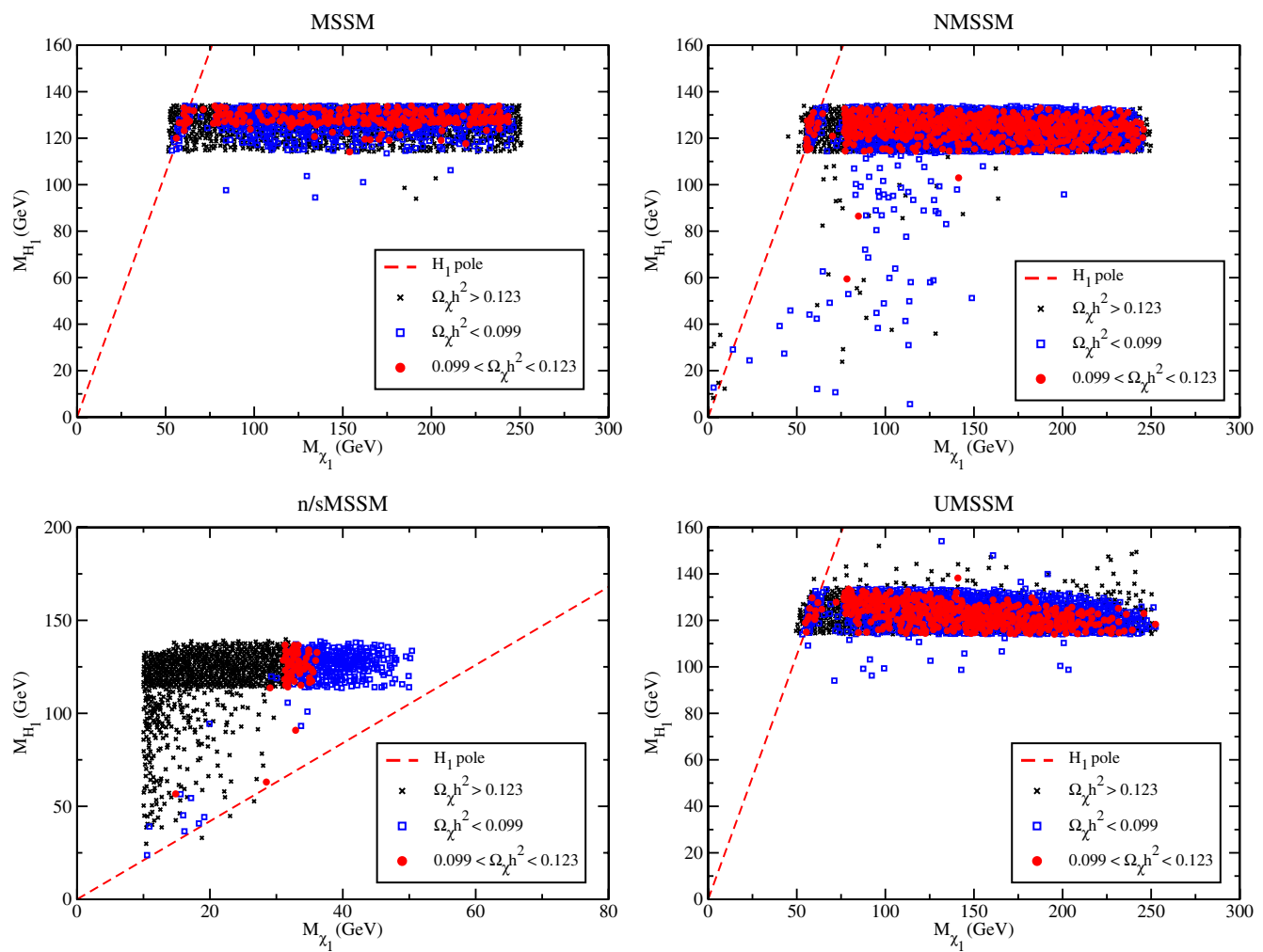

FIG. 3 (color online). Lightest Higgs mass versus lightest neutralino mass for the MSSM, NMSSM, n/sMSSM, and UMSSM. An overdensity of relic neutralinos is denoted by a black $\mathrm{x}$, an underdensity is denoted by a blue open box, and the WMAP observed density is denoted by a red circle.
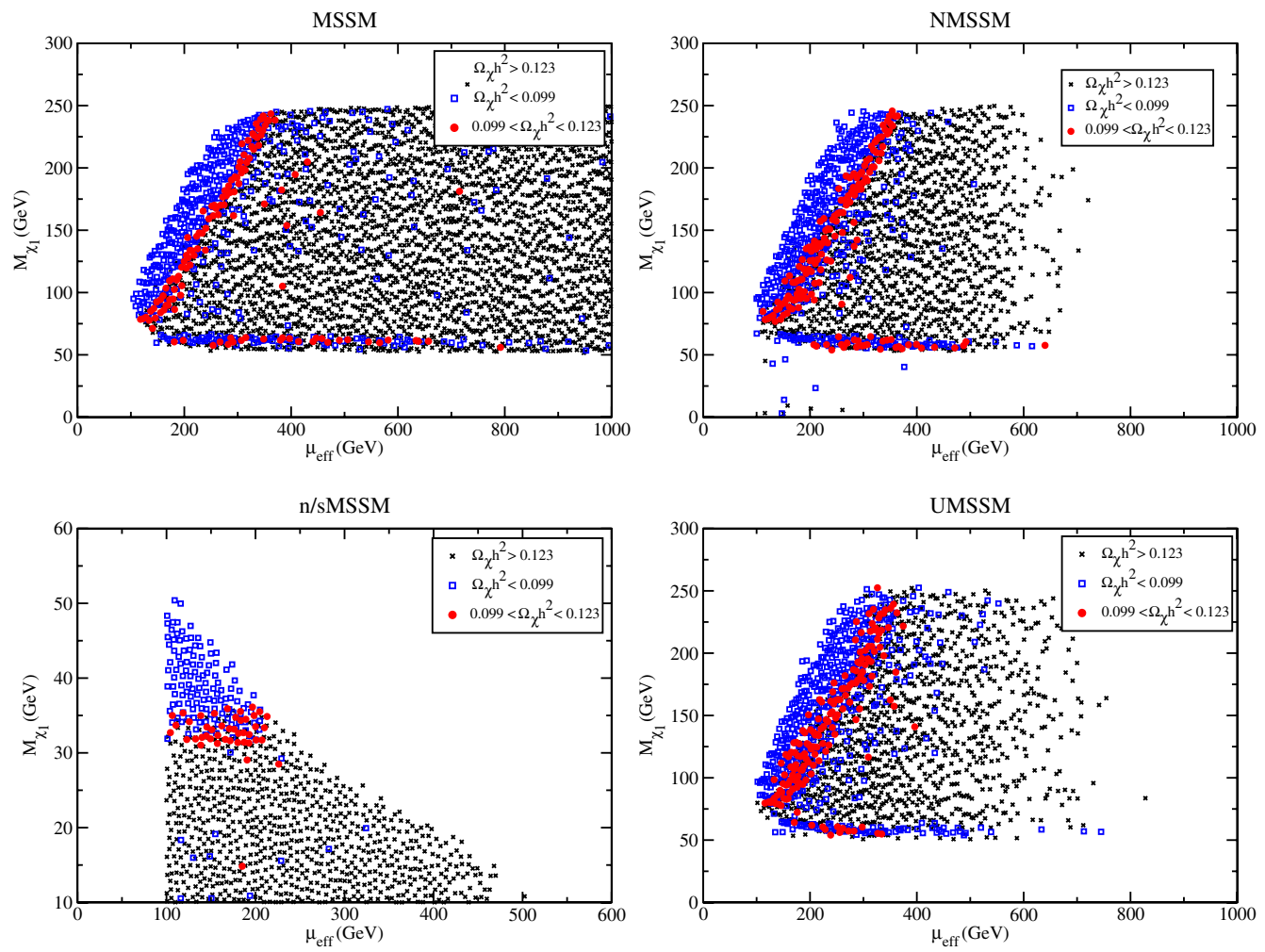

FIG. 4 (color online). Regions of varying relic density in $\mu_{\text {eff }}$ vs $m_{\chi_{1}^{0}}$. The preferred horizontal strip in the MSSM, NMSSM, and UMSSM at $m_{\chi_{1}^{0}} \sim 60 \mathrm{GeV}$ is due to the Higgs pole. The steeply sloped region corresponds to the focus point. 

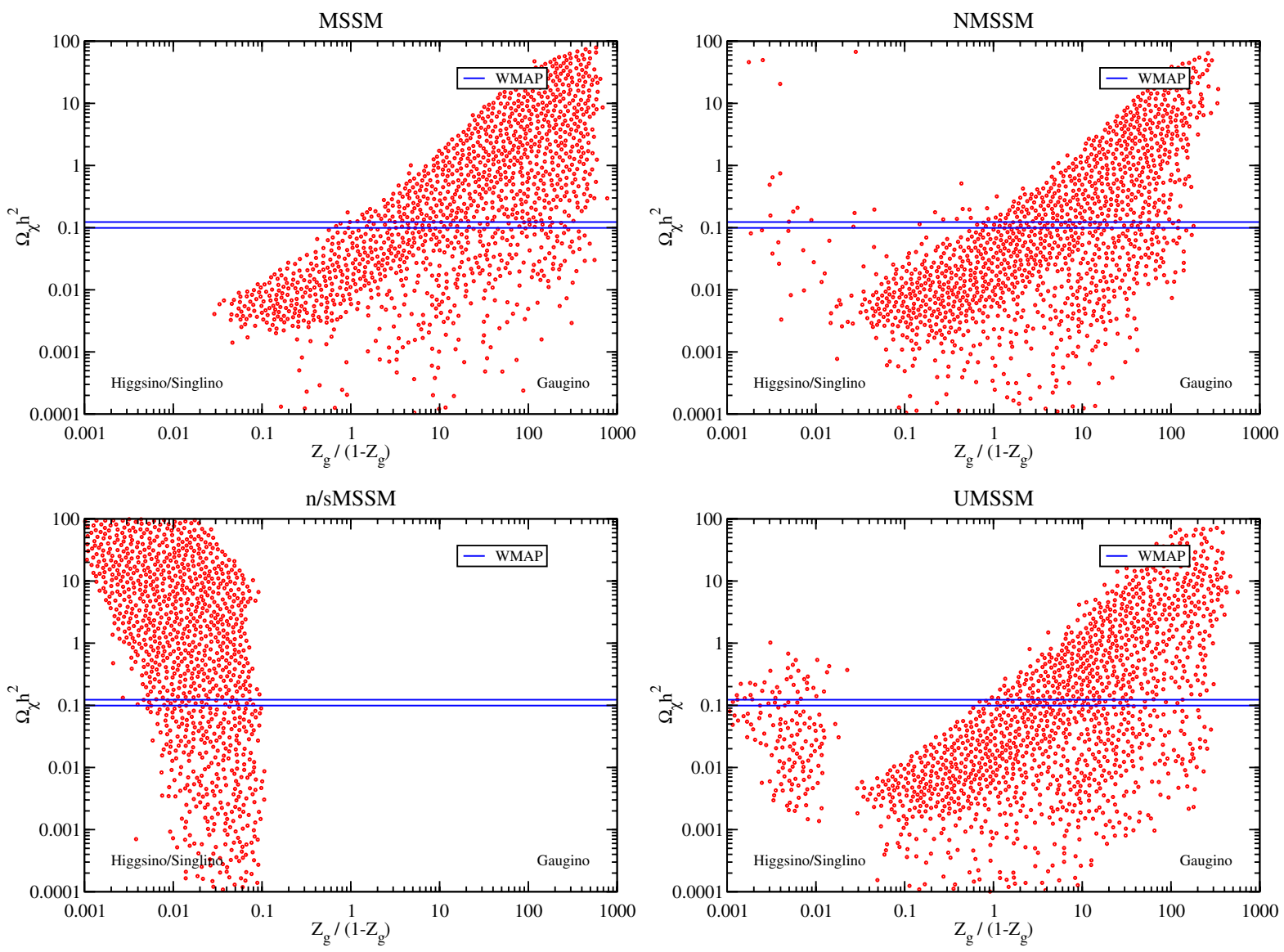

FIG. 5 (color online). Neutralino relic density dependence on the MSSM gaugino fraction $Z_{g}$. Points at the left end of each panel have a lightest neutralino that is dominantly Higgsino and singlino, while at the right side it is dominantly gaugino.

UMSSM. The horizontal band at $M_{\chi_{1}^{0}} \sim 60 \mathrm{GeV}$ is associated with the lightest Higgs pole, while the other band is the focus point region $[93,94]$. This region is characterized by a Higgsino $-B$-ino mixed neutralino which makes neutralino annihilation efficient enough to reproduce the observed relic density [94]. Note that, even though the focus point region requires heavy sleptons and mixed Higgsino dark matter, the neutralino may still coannihilate with neutralinos and charginos of similar mass. Increasing $\mu_{\text {eff }}$ above this region makes the neutralino more gauginolike, which decreases the annihilation rate. However, decreasing $\mu_{\text {eff }}$ will make relic neutralinos annihilate more efficiently, resulting in a relic density below the observed $\Omega_{\mathrm{DM}} h^{2}$ band. The $\mathrm{n} / \mathrm{sMSSM}$ has a neutralino that is dominantly singlino, so the relic density is insensitive to $\mu_{\text {eff }}$.

The gaugino composition of the lightest neutralino is shown in Fig. 5, where $Z_{g}=\left|N_{11}\right|^{2}+\left|N_{12}\right|^{2}$ is the MSSM gaugino fraction. The right sides of the panels indicate a large gaugino fraction while the left sides indicate neutralinos with high Higgsino or singlino/ $Z^{\prime}$-ino composition. In the MSSM, the broad band of WMAP allowed points between $1 \lesssim Z_{g} /\left(1-Z_{g}\right) \lesssim 10$ corresponds to the focus point region while the other scattered points with a higher gaugino fraction are due to the Higgs poles discussed earlier.

In Fig. 6, we show the singlino and $Z^{\prime}$-ino compositions of the neutralino. The UMSSM and n/sMSSM both have a range of singlino/ $Z^{\prime}$-ino fractions that are dominant, but not close to maximal. As the neutralino becomes less MSSMlike, the relic density increases.

Since the coupling between the neutralino pairs and SM particles ( $Z$ and Higgs bosons) needs a suitable value to give an acceptable annihilation rate, the lightest neutralino requires a nonzero MSSM fraction. ${ }^{9}$ In the n/sMSSM, the annihilation is dominated by the $Z$ boson, so the lightest neutralino requires a non-negligible Higgsino component.

The singlino and $Z^{\prime}$-ino dominated neutralino in the UMSSM can also explain the observed relic density. The coupling between the lightest neutralino pair and the singlet dominated $H_{2}$ can be as strong as the $\chi_{1}^{0} \chi_{1}^{0} H_{1}$ coupling in the MSSM; cf. Eq. (B3). The strong coupling and resonant enhancement can yield a large enough annihila-

\footnotetext{
${ }^{9}$ Since we assume that the sleptons are heavy with masses at the $\mathrm{TeV}$ scale, the contributions of $t$-channel diagrams are suppressed.
} 

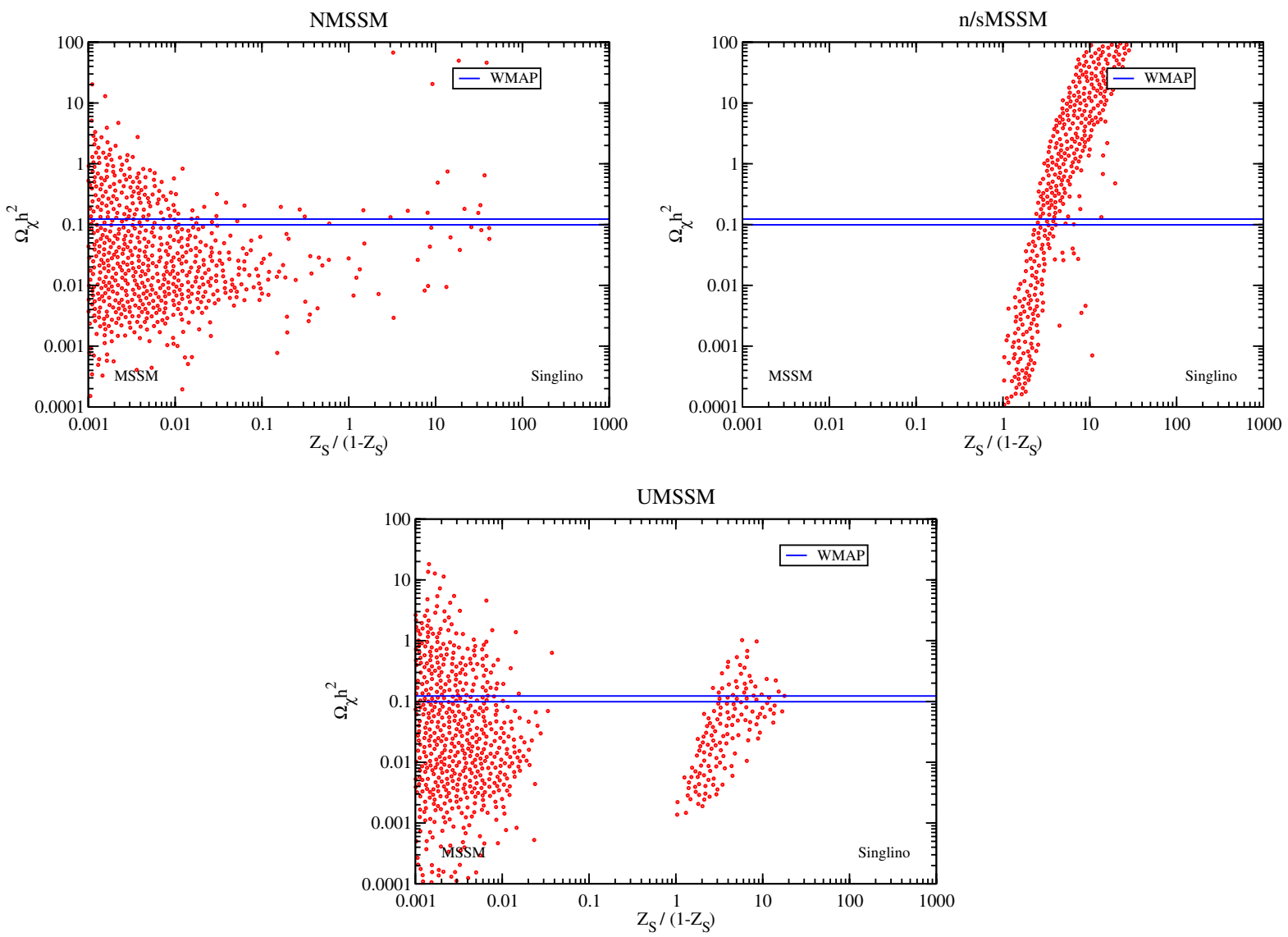

FIG. 6 (color online). Neutralino relic density dependence on the singlino/ $Z^{\prime}$-ino fraction $Z_{S}$. Points at the left end of each panel have a lightest neutralino that is MSSM-like, while at the right side it is dominantly singlino or $Z^{\prime}$-ino.

tion rate to be below the $\Omega_{\mathrm{DM}}$ observation. However, there are parameter points which do fit the observed relic abundance.

\section{III. $\chi_{1}^{0} p \rightarrow \chi_{1}^{0} p$ ELASTIC SCATTERING}

The singlet extended models can give significant changes in the predicted MSSM cross sections relevant to future recoil direct detection experiments. We use the modified version of DARKSUSY discussed in Sec. II to calculate the spin-independent (scalar interaction) and spin-dependent (vector interaction) elastic scattering cross sections of the lightest neutralino off nucleons.

The experimental sensitivity to spin-independent (SI) scattering is much larger since spin-independent processes scatter coherently and therefore are enhanced in scattering from large target nuclei. However, spin-dependent (SD) measurements can be made and have been probed with the Cryogenic Dark Matter Search (CDMS) to the few pb level for elastic proton scattering [95]. The Chicagoland Observatory for Particle Physics (COUPP) experiment which uses superheated $\mathrm{CF}_{3} I$ can greatly improve the limits on the SD processes, down to $10^{-2} \mathrm{pb}$ for proton scattering for a $2 \mathrm{~kg}$ chamber, which is close to the upper cross section expected in the MSSM [96].
Current detection experiments such as EDELWEISS [97] and CDMS have SI sensitivities on the order of $10^{-6} \mathrm{pb}$. In 2007 the sensitivity of the CDMS II experiment is expected to improve to nearly $10^{-8} \mathrm{pb}$ [98]. The proposed future upgrade, SuperCDMS, would reach a detection sensitivity of $10^{-9} \mathrm{pb}$. The WARP experiment with warm liquid Argon is projected to reach a sensitivity of $10^{-10} \mathrm{pb}$ and below; this experiment has just reported first results [99]. The WARP sensitivity from an initial run with $2.3 \mathrm{~L}$ and a total fudicial exposure of $96.5 \mathrm{~kg}$ day are slightly better than that obtained with EDELWEISS. For recent reviews on direct detection experiments, see Refs. [78-80], and for a forthcoming comprehensive summary of the status of direct detection experiments, see Ref. [19].

The SI scattering cross section of a neutralino off a nucleus is given by [84]

$$
\sigma_{\chi_{i}}^{\mathrm{SI}}=\frac{\mu_{\chi_{i}}^{2}}{\pi}\left|Z G_{s}^{p}+(A-Z) G_{s}^{n}\right|^{2}
$$

where $\mu_{\chi_{i}^{0}}=\frac{m_{\chi_{i}^{0}} m_{N}}{m_{\chi_{i}^{0}}+m_{N}}$ is the reduced nucleon-neutralino mass. The parameters $G_{s}^{p}$ and $G_{s}^{n}$ are hadronic matrix elements. Since the cross sections for scattering off protons 
and neutrons are very similar, we focus on scattering from protons, for which the cross section is

$$
\sigma_{\chi p}^{\mathrm{SI}}=\frac{\mu_{\chi p}^{2}}{\pi}\left|G_{s}^{p}\right|^{2}
$$

Here $G_{s}^{p}$ is given in terms of the hadronic matrix elements $\langle N|\bar{q} q| N\rangle$ and couplings by [84]

$$
\begin{aligned}
G_{s}^{p}= & \sum_{q=u, d, s, c, b, t}\langle N|\bar{q} q| N\rangle \frac{1}{2} \sum_{k=1}^{6} \frac{g_{\tilde{q}_{L k} \chi q} g_{\tilde{q}_{R k} \chi q}}{m_{\tilde{q}_{k}}^{2}} \\
& -\sum_{q=u, d, s}\left(\langle N|\bar{q} q| N\rangle \sum_{h=H_{1}, H_{2}, H_{3}} \frac{g_{h \chi \chi} g_{h q q}}{m_{h}^{2}}\right) \\
& -\frac{2}{27} \sum_{q=c, b, t}\left(f_{T G}^{(p)} \frac{m_{p}}{m_{q}} \sum_{h=H_{1}, H_{2}, H_{3}} \frac{g_{h \chi \chi} g_{h q q}}{m_{h}^{2}}\right),
\end{aligned}
$$

where the three terms correspond to the diagrams in Figs. 7(a)-7(c), respectively. We included the following hadronic matrix elements into the DARKSUSY code taken from [100],

$$
\begin{gathered}
\langle N|\bar{q} q| N\rangle=f_{T q}^{p} \frac{m_{p}}{m_{q}}, \\
f_{T u}^{p}=0.020 \pm 0.004, \quad f_{T d}^{p}=0.026 \pm 0.005, \\
f_{T s}^{p}=0.118 \pm 0.062, \quad f_{T G}^{p}=0.84 .
\end{gathered}
$$

We include effects from the exchanges of the scalar quarks of all six generations and the Higgs exchange from light quarks and from gluons via heavy quark loops. The diagrams are shown in Fig. 7. The scalar quark contributions are suppressed by our choice of $\mathrm{TeV}$ squark masses. The uncertainty in the SI scattering cross section is large, of order $60 \%$, due to the above uncertainties in the hadronic matrix elements.

In Fig. 8, we show the predicted direct detection cross section for the MSSM and the singlet extended models along with the sensitivities of EDELWEISS, CDMS II (2005), CDMS 2007, SuperCDMS (25 kg), and WARP $(2.3 \mathrm{~L})$. Over most of the region of neutralino mass, these experiments could find a signal for the MSSM, NMSSM, and UMSSM. There are more points where the lightest neutralino of the MSSM may be detectable via recoil experiments than typical CMSSM expectations since the general parameter treatment of the MSSM can yield scattering cross sections that are a few orders of magnitude larger than in the CMSSM [78-80]. The experimental sensitivities shown assume a local dark matter density of $\rho=0.3 \mathrm{GeV} / \mathrm{cm}^{3}$. Therefore, the sensitivities only apply for the points in which the relic density is saturated by neutralinos (red points).

Many of the points consistent with the observed relic density are within the reach of SuperCDMS and WARP $(2.3 \mathrm{~L})$. The region below the WARP sensitivity corresponds to neutralino annihilation through the $H_{1}\left(\right.$ or $\left.H_{2}\right)$ pole. To account for the observed relic density, the $\chi_{1}^{0} \chi_{1}^{0} H_{i}$ coupling is small to balance the resonant enhancement of the annihilation rate. This forces the scattering cross section via the Higgs states to be small, and thus fall below future sensitivities.

Since the n/sMSSM has a light singlino state, the strict limits on the allowed neutralino mass in this model from the expected relic density can be used to deduce a lower limit on the direct detection cross section. This is apparent in Fig. 8(c) as most of the allowed region is above the expected sensitivity of CDMS 2007, which should allow CDMS to place extremely strong constraints on this model if no signal is found, assuming that the neutralino is responsible for a major part of the dark matter in the Universe. Still, detection could be precluded if annihilation through the Higgs resonance occurs with $m_{\chi_{1}^{0}}<30 \mathrm{GeV}$ and $M_{H_{1}} \simeq m_{\chi_{1}^{0}} / 2$, as shown by the isolated low $\Omega_{\chi_{1}^{0}} h^{2}$ points in Fig. 1(c), or by neutralino decays into an additional, still lighter particle [92].

The predictions of the SI cross sections are heavily dependent on the local density of dark matter in our Galaxy. The present halo model has many assumptions and uncertainties. Caustic rings are predicted to exist in the Galaxy with a ring overlapping in the vicinity of our

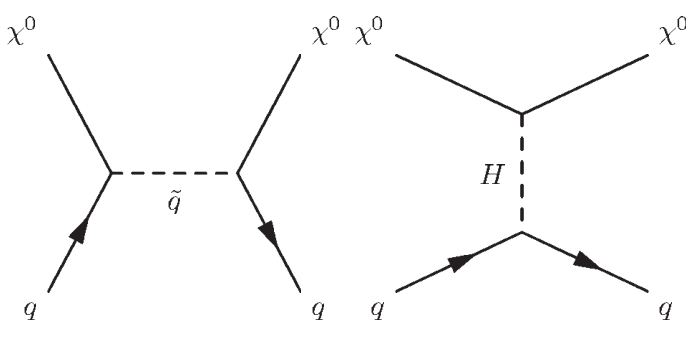

(a) (b)

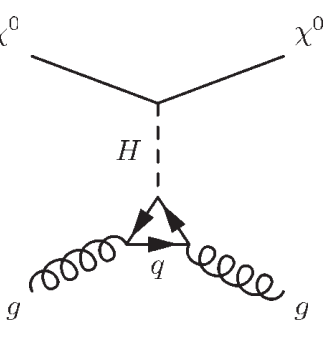

(c)

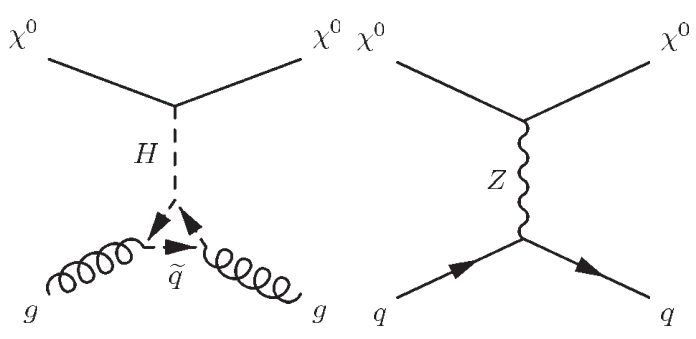

(d)

(e)

FIG. 7. Diagrams contributing to neutralino-hadron scattering. Scalar quark (a), Higgs boson (b), and $Z$ boson (e) exchange contribute to the SI scattering cross section. A significant contribution also comes from the Higgs scattering off gluons via heavy quark and squark loops (c), (d). Processes which contribute to the SD scattering include squark exchange (a) and $Z$ boson exchange (e). However, SD predictions are far less constrained than SI. 


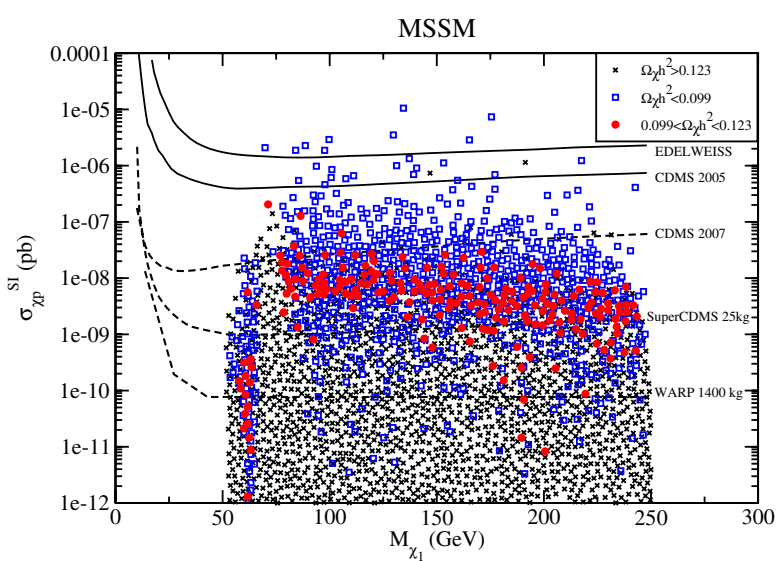

(a)

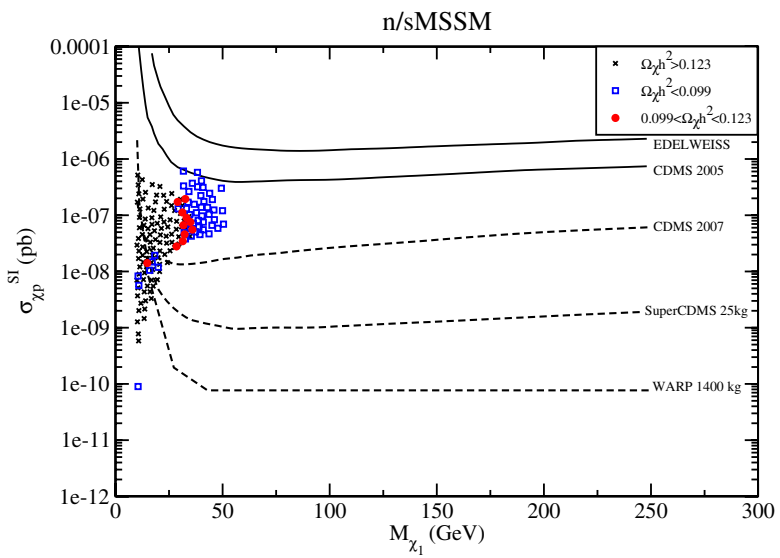

(c)

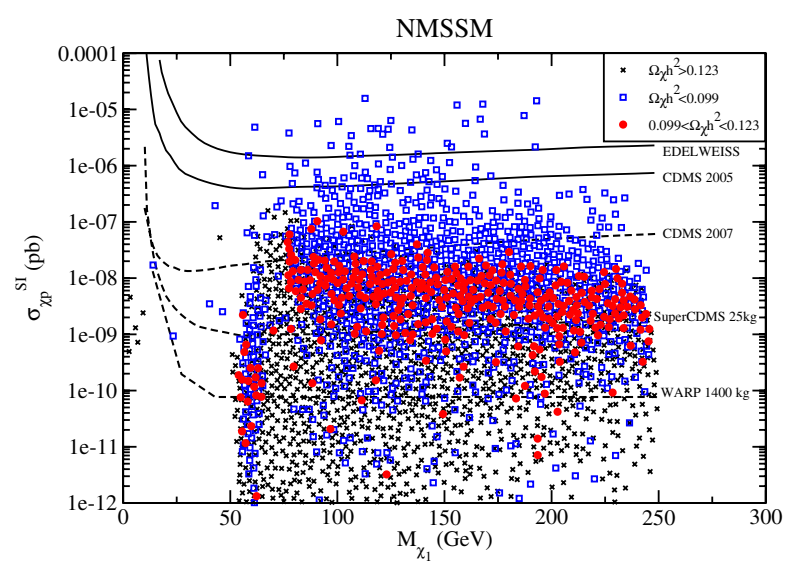

(b)

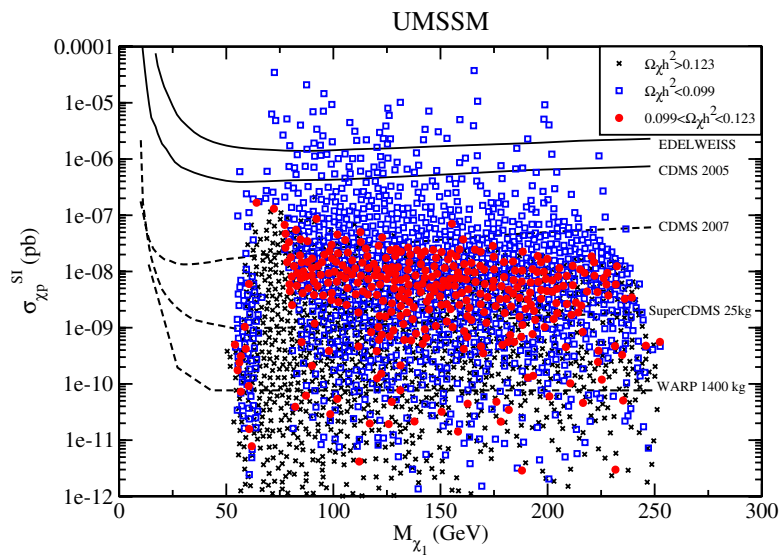

(d)

FIG. 8 (color online). Expected SI direct detection cross section for (a) MSSM, (b) NMSSM, (c) n/sMSSM, and (d) UMSSM. The expected sensitivities of the EDELWEISS, CDMS II (2005), CDMS 2007, SuperCDMS (25 kg), and WARP (2.3 L) experiments are shown. Over most of the neutralino mass range, the experiments should detect the signals from the MSSM, NMSSM, and UMSSM. However, if the neutralino annihilates via a Higgs boson resonance, the relic density may be in the preferred region while the direct detection rate is out of the reach of future experiments. The experimental sensitivities shown assume a local dark matter density of $\rho=0.3 \mathrm{GeV} / \mathrm{cm}^{3}$. Therefore, the sensitivities only apply for the points in which the relic density is saturated by neutralinos (red circles).

solar system [101]. That could increase the local dark matter velocity by a factor of 3 or more, resulting in a correspondingly enhanced flux and direct detection rate.

\section{CONCLUSIONS}

In our investigation of the relic density constraints and the direct detection capabilities of present and upcoming dark matter experiments, we modified the DARKSUSY code to include the additional couplings and processes of singlet extended MSSM models. ${ }^{10}$ We also updated the DARKSUSY values for the hadronic matrix elements and experimental constraints. Our analysis assumed multi-TeV masses of squarks and sleptons. We generally found close similarities

\footnotetext{
${ }^{10}$ Lighter squarks and sleptons would allow the possibility of additional coannihilation regions.
}

of the NMSSM and UMSSM predictions with those of the MSSM due to the similarity of the compositions of their light neutralino states. However, the n/sMSSM showed exceptional differences due to very light neutralinos that are dominantly singlino. Some notable results from our study include the following:

(i) The observed relic density can be accounted for in all of the singlet extended models, mainly with mixed Higgsino-gaugino dark matter. In some cases, the lightest neutralino is dominantly singlino, or a mixture of singlino and $Z^{\prime}$-ino in the case of the UMSSM. The predicted relic density can also match the observed value if the neutralinos annihilate through a Higgs boson resonance. The annihilation via the lightest Higgs boson can enhance the rate sufficiently to yield the correct relic density, even though the neutralino is typically $B$-ino in this case. 
(ii) The neutralino in the n/sMSSM typically has a mass in the range $30 \mathrm{GeV} \lesssim m_{\chi_{1}^{0}} \lesssim 37 \mathrm{GeV}$ to account for the total relic density found by the WMAP Collaboration. The upper bound on $m_{\chi_{1}^{0}}$ may extend to $50 \mathrm{GeV}$ if the neutralino relic density is below the observed DM value $[38,53,83]$. Masses smaller than $30 \mathrm{GeV}$ may be allowed if annihilation through the light $C P$-odd Higgs boson or neutralino decays occurs to a still lighter state.

(iii) The singlino/ $Z^{\prime}$-ino dominated neutralino in the UMSSM can account for the observed relic density. Since the coupling between the lightest neutralino pair and the singlet dominated $\mathrm{H}_{2}$ can be large and resonant enhancement of the annihilation cross section via $\mathrm{H}_{2}$ can occur, the relic density can fall into the observed $2 \sigma$ range.

(iv) The MSSM, NMSSM, and UMSSM predict spinindependent proton scattering cross sections that may be detectable at SuperCDMS and WARP and be consistent with the WMAP $\Omega_{\mathrm{DM}}$ measurements. However, the recoil predictions of some models may be undetectable by these experiments due to the small Higgs neutralino coupling.

(v) The n/sMSSM SI proton scattering cross sections are highly favored to be detectable at CDMS 2007 while being compatible with WMAP $\Omega_{\mathrm{DM}}$ observations. However, if neutralino annihilation occurs through a light Higgs, measurement of the scattering cross section can fall below the sensitivities of future experiments. (vi) Our MSSM predictions are more general than mSUGRA results.

In addition to these constraints from the relic density bounds, our study of further constraints in the appendixes found

(i) Perturbativity constraints on $\lambda$ from RGE evolution give upper bounds on $\lambda\left(M_{t}\right)$ below unity and require $\tan \beta \gtrsim 1.9$.

(ii) Requiring that the xMSSM models are consistent with the anomalous magnetic moment of the muon, $\Delta a_{\mu}$, provides lower limits of $\tan \beta \geqslant 5$, though there are still theoretical uncertainties.

\section{ACKNOWLEDGMENTS}

We thank $\mathrm{H}$. Baer for valuable conversations about the capabilities of future direct detection experiments. This work was supported in part by the U.S. Department of Energy under Grant No. DE-FG02-95ER40896, by the Wisconsin Alumni Research Foundation, by the Friends of the IAS, and by the National Science Foundation Grant No. PHY-0503584.

\section{APPENDIX A: NEUTRALINO MASS MATRIX}

The neutralino mass matrix of the singlet extended models is extended due to the additional singlino and $Z^{\prime}$-ino states that mix with the MSSM gauginos and Higgsinos. In the $\left(\tilde{B}^{0}, \tilde{W}^{0}, \tilde{H}_{d}^{0}, \tilde{H}_{u}^{0}, \tilde{S}^{0}, \tilde{Z}^{\prime 0}\right)$ basis, the mass matrix is

$$
\mathbf{M}_{\chi^{0}}=\left(\begin{array}{cccccc}
M_{1} & 0 & -g_{1} v_{1} / 2 & g_{1} v_{2} / 2 & 0 & 0 \\
0 & M_{2} & g_{2} v_{1} / 2 & -g_{2} v_{2} / 2 & 0 & 0 \\
-g_{1} v_{2} / 2 & g_{2} v_{1} / 2 & 0 & -\mu_{\mathrm{eff}} & -\mu_{\mathrm{eff}} \boldsymbol{v}_{2} / s & g_{Z^{\prime}} Q_{H_{1}}^{\prime} v_{1} \\
g_{1} v_{2} / 2 & -g_{2} v_{2} / 2 & -\mu_{\mathrm{eff}} & 0 & -\mu_{\mathrm{eff}} v_{1} / s & g_{Z^{\prime}} Q_{H_{2}}^{\prime} v_{2} \\
0 & 0 & -\mu_{\mathrm{eff}} \boldsymbol{v}_{2} / s & -\mu_{\mathrm{eff}} \boldsymbol{v}_{1} / s & \sqrt{2} \kappa s & g_{Z^{\prime}} Q_{S}^{\prime} s \\
0 & 0 & g_{Z^{\prime}} Q_{H_{1}}^{\prime} v_{1} & g_{Z^{\prime}} Q_{H_{2}}^{\prime} \boldsymbol{v}_{2} & g_{Z^{\prime}} Q_{S}^{\prime} s & M_{1^{\prime}} s
\end{array}\right)
$$

where the $M_{i}$ are the gaugino masses (assumed universal at the GUT scale), and $v_{1}, v_{2}$, and $s$ are, respectively, $\sqrt{2}\left(\left\langle H_{d}\right\rangle,\left\langle H_{u}\right\rangle,\langle S\rangle\right)$.

\section{APPENDIX B: XMSSM COUPLINGS}

Couplings of the singlet extended models are changed with respect to the MSSM due to the additional singlet and $Z^{\prime}$ contributions.

(i) The Yukawa, $C P$-even, and $C P$-odd Higgs couplings are

$$
g_{d d H_{i}}=g_{f f h}^{\mathrm{SM}} \frac{R_{+}^{i 1}}{\cos \beta}, \quad g_{u u H_{i}}=g_{f f h}^{\mathrm{SM}} \frac{R_{+}^{i 2}}{\sin \beta},
$$

$$
g_{d d A_{i}}=i \gamma^{5} g_{f f h}^{\mathrm{SM}} \frac{R_{-}^{i 1}}{\cos \beta}, \quad g_{\text {uи }}=i \gamma^{5} g_{f f h}^{\mathrm{SM}} \frac{R_{-}^{i 2}}{\sin \beta},
$$

where $R_{+}^{i j}$ and $R_{-}^{i j}$ are the rotation matrices that diagonalize the $C P$-even and $C P$-odd Higgs masssquared matrices, respectively, in the $\left(H_{u}, H_{d}, S\right)$ basis, and $g_{f f h}^{\mathrm{SM}}$ are the corresponding couplings in the SM (see Ref. [37]).

There are some couplings that do not have a standard model counterpart, as follows:

(ii) The Higgs-neutralino-neutralino coupling constants are 


$$
\begin{aligned}
g_{H_{i} \chi_{1}^{0} \chi_{1}^{0}}= & {\left[\left(g_{1} N_{11}-g_{2} N_{12}-g_{1^{\prime}} Q_{H_{d}} N_{16}\right) N_{13}\right.} \\
& \left.+\sqrt{2} \lambda N_{14} N_{15}\right] R_{+}^{i 1}+\left[\left(g_{2} N_{12}-g_{1} N_{11}\right.\right. \\
& \left.\left.-g_{1^{\prime}} Q_{H_{u}} N_{16}\right) N_{14}+\sqrt{2} \lambda N_{13} N_{15}\right] R_{+}^{i 2} \\
& +\left[-g_{1^{\prime}} Q_{S} N_{16} N_{15}+\sqrt{2} \lambda N_{13} N_{14}\right. \\
& \left.-\sqrt{2} \kappa N_{15} N_{15}\right] R_{+}^{i 3}, \\
g_{A_{i} \chi_{1}^{0} \chi_{1}^{0}}= & i \gamma^{5}\left[\left(g_{1} N_{11}-g_{2} N_{12}-g_{1^{\prime}} Q_{H_{d}} N_{16}\right) N_{13}\right. \\
& \left.+\sqrt{2} \lambda N_{14} N_{15}\right] R_{-}^{i 1}+\left[\left(g_{2} N_{12}-g_{1} N_{11}\right.\right. \\
& \left.\left.-g_{1^{\prime}} Q_{H_{u}} N_{16}\right) N_{14}+\sqrt{2} \lambda N_{13} N_{15}\right] R_{-}^{i 2} \\
& +\left[-g_{1^{\prime}} Q_{S} N_{16} N_{15}+\sqrt{2} \lambda N_{13} N_{14}\right. \\
& \left.-\sqrt{2} \kappa N_{15} N_{15}\right] R_{-}^{i 3},
\end{aligned}
$$

where $N_{i j}$ is the rotation matrix that diagonalizes the neutralino mass matrix in Eq. (A1).

(iii) The left- and right-handed neutralino-quark-squark coupling constants are

$$
\begin{aligned}
& g_{L \tilde{u}_{k} \chi^{0} u_{i}}=-\frac{Z_{\tilde{u}}^{i k *}}{\sqrt{2}}\left(\frac{1}{3} N_{11} g_{1}+N_{12} g_{2}\right) \\
&-Y_{u}^{i} Z_{\tilde{u}}^{(i+3) k *} N_{14}, \\
& g_{R \tilde{u}_{k} \chi^{0} u_{i}}=\frac{2 \sqrt{2} g_{2}}{3} Z_{\tilde{u}}^{(i+3) k *} N_{11}^{*}-Y_{u}^{i} Z_{\tilde{u}}^{i k *} N_{14}^{*}, \\
& g_{L \tilde{d}_{k} \chi^{0} d_{i}}=-\frac{Z_{\tilde{d}}^{i k}}{\sqrt{2}}\left(\frac{1}{3} N_{11} g_{1}-N_{12} g_{2}\right) \\
&-Y_{d}^{i} Z_{\tilde{d}}^{(i+3) k} N_{13}, \\
& g_{R \tilde{d}_{k} \chi^{0} d_{i}}= \frac{-\sqrt{2} g_{2}}{3} Z_{\tilde{d}}^{(i+3) k} N_{11}^{*}-Y_{d}^{i} Z_{\tilde{d}}^{i k} N_{13}^{*},
\end{aligned}
$$

where $Z_{\tilde{q}}^{i k}$ and $Z_{\tilde{q}}^{(i+3) k}$ are the rotation matrices for the left- and right-handed scalar quarks, respectively. The Yukawa couplings, $Y_{q}^{i}$, are defined for quark $q_{i}$.

(iv) The trilinear Higgs coupling is modified due to the additional singlet state in the nMSSM, NMSSM, and UMSSM. The $H_{i} A_{j} A_{k}$ coupling can be found using the projection [37]

$$
C_{H_{i} A_{j} A_{k}}=P_{H_{i}} P_{A_{j}} P_{A_{k}} V,
$$

where $V$ is the Higgs potential and the projection operators are

$$
P_{H_{j}}=\frac{1}{\sqrt{2}}\left(R_{+}^{j 1} \frac{\partial}{\partial \phi_{d}}+R_{+}^{j 2} \frac{\partial}{\partial \phi_{u}}+R_{+}^{j 3} \frac{\partial}{\partial \sigma}\right),
$$

$$
P_{A_{k}}=\frac{1}{\sqrt{2}}\left(R_{-}^{k 1} \frac{\partial}{\partial \varphi_{d}}+R_{-}^{k 2} \frac{\partial}{\partial \varphi_{u}}+R_{-}^{k 3} \frac{\partial}{\partial \xi}\right) .
$$

In a similar manner the $H_{i} H_{j} H_{k}$ coupling is

$$
C_{H_{i} H_{j} H_{k}}=P_{H_{i}} P_{H_{j}} P_{H_{k}} V \text {, }
$$

and the $H_{i} H_{j} A_{k}$ coupling is

$$
C_{H_{i} H_{j} A_{k}}=P_{H_{i}} P_{H_{j}} P_{A_{k}} V .
$$

\section{APPENDIX C: EXPERIMENTAL CONSTRAINTS}

We generated the Higgs and neutralino masses and couplings by scanning over the parameters given in Ref. [37] and diagonalizing the Higgs and neutralino mass matrices. For each model, the following LEP experimental constraints are applied: The $Z Z h$ coupling limits, the bound on the lightest chargino, the charged Higgs mass bound, the $A h$ associated production search limits and the contribution to the invisible decay width of the $Z$ boson, and the limit on the $Z-Z^{\prime}$ mixing in the UMSSM, as in Ref. [37]. In addition, we considered limits from the anomalous magnetic moment of the muon, and coupling perturbativity constraints. We also considered constraints from the branching fraction of $b \rightarrow s \gamma$ [102] and found that the distribution of allowed points was not qualitatively changed; the plots shown do not include this constraint.

\section{Anomalous magnetic moment of the muon}

The experimentally measured value of the anomalous magnetic moment of the muon $a_{\mu}=(g-2)_{\mu} / 2$ shows a $3.4 \sigma$ deviation $[103,104]$

$$
\Delta a_{\mu}=a_{\mu}(\exp )-\mathrm{a}_{\mu}(\mathrm{SM})=(27.6 \pm 8.1) \times 10^{-10}
$$

from the standard model prediction based on the use of

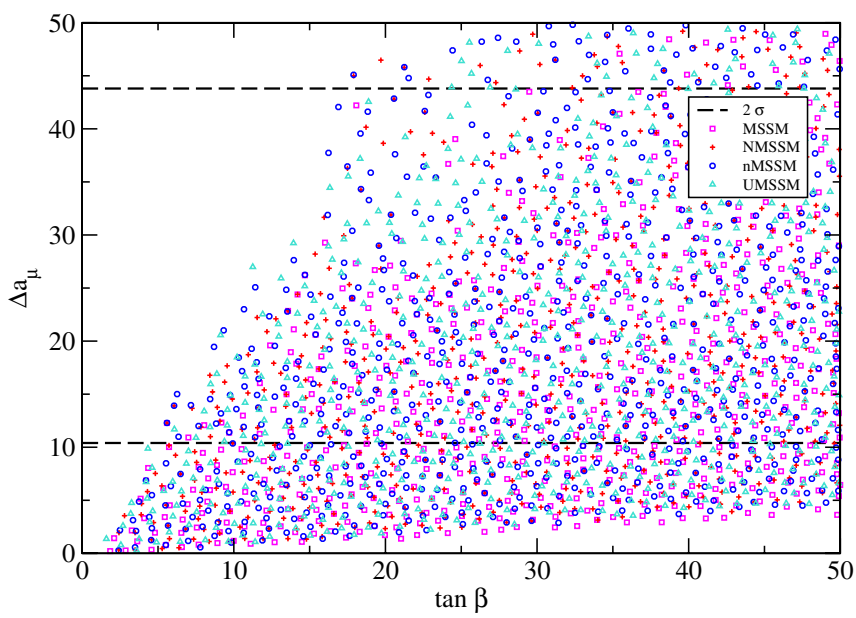

FIG. 9 (color online). The deviation of the anomalous magnetic moment of the muon $\Delta a_{\mu}$ consistent with the $2 \sigma$ uncertainty for values of $\tan \beta$ in various models is within the dashed horizontal lines. 
$e^{+} e^{-} \rightarrow \pi \pi$ data from CMD-2 and KLOE. The constraints on the UMSSM parameter space due to the $a_{\mu}$ deviation were previously studied in Ref. [105]. We apply the above $a_{\mu}$ constraint here and find that the results do not vary significantly across models as shown in Fig. 9. In particular, $\Delta a_{\mu}$ gives modest lower limits on $\tan \beta$ when using the parameter values in Ref. [37] in a scan over the soft slepton masses $300 \mathrm{GeV} \leq M_{\tilde{L}}=M_{\tilde{E}} \leq 2 \mathrm{TeV}$. At the $2 \sigma$ level the $\Delta a_{\mu}$ bound limits $\tan \beta \gtrsim 5$. We did not impose this constraint in our analyses, because there are still theoretical uncertainties involving the disagreement between estimates using $e^{+} e^{-}$and $\tau$ decay data $[103,104]$.

\section{Perturbativity constraints}

The singlet models that we consider may have gauge coupling unification at a grand unification scale $M_{\mathrm{GUT}} \sim$ $2 \times 10^{16} \mathrm{GeV}$. We may require that the gauge and Yukawa couplings remain perturbative up to this scale. To impose this constraint, we evaluate the running of the couplings in each model according to the renormalization group equations (RGEs) [48,60-62,77,106-112] at one-loop order:

$$
\begin{aligned}
\frac{d g_{i}}{d t}= & \beta_{i} g_{i}^{3}, \quad \frac{d g_{1^{\prime}}}{d t}=\beta_{1^{\prime}} g_{1^{\prime}}^{3}, \quad \frac{d \kappa}{d t}=6 \kappa\left[\lambda^{2}+\kappa^{2}\right] \\
\frac{d \lambda}{d t}= & \lambda\left[4 \lambda^{2}+2 \kappa^{2}+3 h_{t}^{2}-3 g_{2}^{2}-\frac{3}{5} g_{1}^{2}\right. \\
& \left.-2 g_{1^{\prime}}^{2}\left(Q_{S}^{2}+Q_{H_{d}}^{2}+Q_{H_{u}}^{2}\right)\right] \\
\frac{d h_{t}}{d t}= & h_{t}\left[\lambda^{2}+6 h_{t}^{2}-\frac{16}{3} g_{3}^{2}-3 g_{2}^{2}-\frac{13}{15} g_{1}^{2}\right. \\
& \left.-2 g_{1^{\prime}}^{2}\left(Q_{u}^{2}+Q_{Q}^{2}+Q_{H_{u}}^{2}\right)\right]
\end{aligned}
$$

where $t=\frac{1}{(4 \pi)^{2}} \ln \left(\mu / m_{t}\right)$. Here $h_{t}$ is the top quark Yukawa coupling and $Q_{H_{u}}, Q_{H_{d}}, Q_{S}, Q_{u}$, and $Q_{Q}$ are the $U(1)^{\prime}$ charges of the up and down Higgs doublets, the Higgs singlet, the up type quark, and the quark doublet, respectively. For specific models, the parameters $g_{1}^{\prime}$ and $\kappa$ are appropriately turned off.

The scale factors and $\beta$ functions are given in Table II. ${ }^{11}$ In Table III we give the quantum numbers under the standard model gauge groups, $U(1)_{\chi}$ and $U(1)_{\psi}$ (symmetries arising when the $E_{6}$ model is broken [106]), and $U(1)^{\prime}$ (the additional symmetry of interest in the UMSSM). As $U(1)^{\prime}$ is simply a linear combination of $U(1)_{\chi}$ and $U(1)_{\psi}$, the quantum numbers under this symmetry are given by

$$
Q=Q_{\chi} \cos \theta_{E 6}+Q_{\psi} \sin \theta_{E 6}
$$

and thus depend on the particular value of $\theta_{E 6}$.

The RGEs were evolved from the low scale, chosen to be $m_{t}=171 \mathrm{GeV}$, up to $M_{\mathrm{GUT}} \sim 2 \times 10^{16} \mathrm{GeV}$. The gauge

\footnotetext{
${ }^{11}$ We do not include the effect of kinetic mixing in the UMSSM [113].
}

TABLE II. One-loop $\beta$ functions for the three models $[106,107]$. The traces involve sums over all matter superfields and generations.

\begin{tabular}{lcccc}
\hline \hline & $\beta_{1}$ & $\beta_{2}$ & $\beta_{3}$ & $\beta_{1^{\prime}}$ \\
\hline NMSSM/nMSSM & $33 / 5$ & 1 & -3 & 0 \\
UMSSM & $\frac{3}{5} \operatorname{Tr} Q_{Y}^{2}=9+\frac{3}{5}$ & 4 & 0 & $\operatorname{Tr} Q^{2}=9+4 Q_{H_{u}}^{2}$ \\
\hline \hline
\end{tabular}

TABLE III. Matter multiplets in the UMSSM and their various quantum numbers (according to [106], but noting our difference in normalization). The index $i$ sums over generations.

\begin{tabular}{lccc|ccc}
\hline \hline Superfields & $S U(3)_{c}$ & $S U(2)_{L}$ & $Q_{Y}=\sqrt{\frac{5}{3}} Q_{1}$ & $U(1)^{\prime}$ & $\sqrt{40} Q_{\chi}$ & $\sqrt{24} Q_{\psi}$ \\
\hline$\hat{Q}_{i}$ & $\mathbf{3}$ & $\mathbf{2}$ & $\frac{1}{6}$ & $Q_{Q}$ & -1 & 1 \\
$\hat{u}_{i}^{c}$ & $\mathbf{3}$ & $\mathbf{1}$ & $-\frac{2}{3}$ & $Q_{u}$ & -1 & 1 \\
$\hat{d}_{i}^{c}$ & $\mathbf{3}$ & $\mathbf{1}$ & $\frac{1}{3}$ & $Q_{d}$ & 3 & 1 \\
$\hat{L}_{i}$ & $\mathbf{1}$ & $\mathbf{2}$ & $-\frac{1}{2}$ & $Q_{L}$ & 3 & 1 \\
$\hat{E}_{i}^{c}$ & $\mathbf{1}$ & $\mathbf{1}$ & 1 & $Q_{E}$ & -1 & 1 \\
$\hat{H}_{1 i}$ & $\mathbf{1}$ & $\mathbf{2}$ & $-\frac{1}{2}$ & $Q_{H_{d}}$ & -2 & -2 \\
$\hat{H}_{2 i}$ & $\mathbf{1}$ & $\mathbf{2}$ & $\frac{1}{2}$ & $Q_{H_{u}}$ & 2 & -2 \\
$\hat{D}_{i}$ & $\mathbf{3}$ & $\mathbf{1}$ & $-\frac{1}{3}$ & $Q_{D}$ & 2 & -2 \\
$\hat{\bar{D}}_{i}$ & $\mathbf{3}$ & $\mathbf{1}$ & $\frac{1}{3}$ & $Q_{\bar{D}}$ & -2 & -2 \\
$\hat{S}_{i}$ & $\mathbf{1}$ & $\mathbf{1}$ & 0 & $Q_{S}$ & 0 & 4 \\
$\hat{N}_{i}^{c}$ & $\mathbf{1}$ & $\mathbf{1}$ & 0 & $Q_{N}$ & -5 & 1 \\
$\hat{H}_{3}$ & $\mathbf{1}$ & $\mathbf{2}$ & $-\frac{1}{2}$ & $-Q_{H_{u}}$ & -2 & 2 \\
$\hat{\bar{H}}_{3}$ & $\mathbf{1}$ & $\mathbf{2}$ & $\frac{1}{2}$ & $Q_{H_{u}}$ & 2 & -2 \\
\hline \hline
\end{tabular}

TABLE IV. The maximum allowable value of $\lambda$ for perturbativity in the NMSSM, as a function of $\kappa$.

\begin{tabular}{lccccc}
\hline \hline$\kappa$ & 0.0 & 0.3 & 0.4 & 0.5 & 0.6 \\
\hline$\lambda_{\max }$ & 0.70 & 0.64 & 0.58 & 0.47 & 0.25 \\
\hline \hline
\end{tabular}

couplings were first run independently up to $m_{t}$ from $M_{Z}$, using [75,114]

$$
\begin{aligned}
\sin ^{2} \theta_{W}\left(M_{Z}\right) & =0.23122 \pm 0.00015 \alpha, \\
\left(M_{Z}\right) & =1 /(127.918 \pm 0.018) \alpha_{S}, \\
\left(M_{Z}\right) & =0.1189 \pm 0.0010
\end{aligned}
$$

where $\alpha=e^{2} / 4 \pi, \alpha_{S}=g_{3}^{2} / 4 \pi, g_{1}=\sqrt{\frac{5}{3}} g_{Y}=\sqrt{\frac{5}{3}} e / \cos \theta_{W}$, and $g_{2}=e / \sin \theta_{W}$. The gauge couplings were assumed to unify at the GUT scale, with the canonical normalization $g_{1^{\prime}}=g_{1}=g_{2}=g_{3}$ at the unification scale. ${ }^{12}$ From the top quark pole mass, we obtained the low-scale value of the top quark Yukawa coupling

$$
h_{t}\left(m_{t}\right)=\frac{m_{t}\left(m_{t}\right)}{\frac{1}{\sqrt{2}} v \sin \beta} .
$$

\footnotetext{
${ }^{12} \beta_{1^{\prime}}$ depends weakly on $\theta_{E_{6}}$, which is scanned, because of the $\hat{H}_{3}, \hat{\bar{H}}_{3}$ pair needed for gauge unification [106]. This implies that $g_{1^{\prime}}=\sqrt{\lambda_{g}} g_{1}$ at the weak scale, where $\lambda_{g}$, which depends on $\theta_{E_{6}}$, is typically within $6 \%$ of unity.
} 


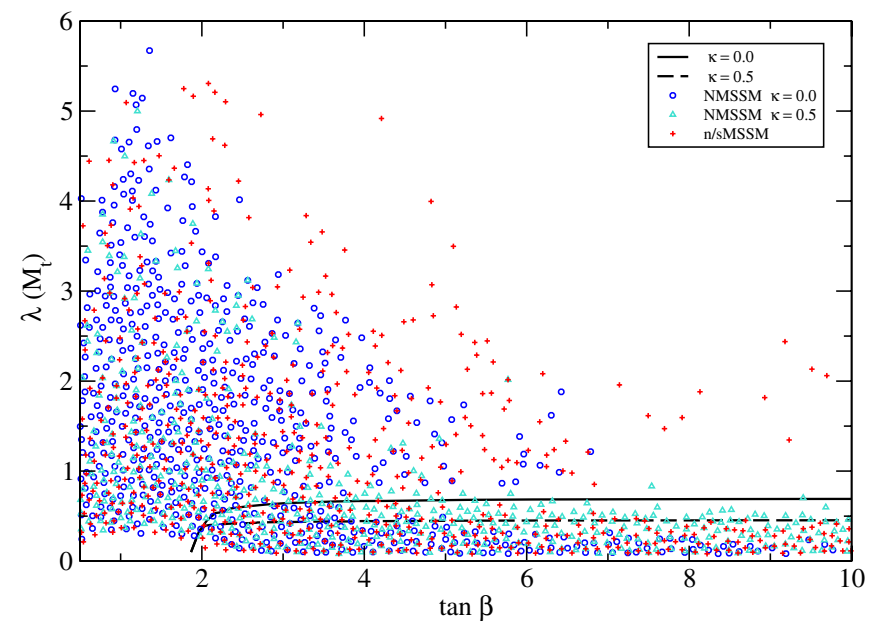

FIG. 10 (color online). Values of $\lambda$ vs $\tan \beta$ consistent with all considered constraints except perturbativity in scans over all parameters. The solid and dashed lines represent the maximum allowed values of $\lambda\left(m_{t}\right)$ that remain perturbative in the NMSSM for $\kappa=0$ and $\kappa=0.5$. The $\mathrm{n} / \mathrm{sMSSM}$ constraint is given by the $\kappa=0$ result of the NMSSM.

For the couplings to remain perturbative up to $M_{\mathrm{GUT}}$, we require

$$
\kappa^{2} \leq 4 \pi ; \quad \lambda^{2} \leq 4 \pi ; \quad h_{t}^{2} \leq 4 \pi .
$$

The NMSSM perturbativity constraints are shown in Fig. 10 as a solid line for $\kappa=0$ and a dashed line for $\kappa=$ 0.5 . The $\mathrm{n} / \mathrm{sMSSM}$ corresponds to the $\kappa=0$ limit of the NMSSM. There are many points with large values of $\lambda$ at low $\tan \beta$ that violate the limit in the $\kappa=0$ NMSSM and $\mathrm{n} /$ sMSSM. Figure 10 also demonstrates that perturbativity becomes slightly more constraining in the NMSSM for larger $\kappa$, as $\kappa$ contributes to the running of $\lambda$. The maximum allowed values of $\lambda\left(m_{t}\right)$ for moderate values of $\tan \beta$ are given in Table IV for several values of $\kappa$. These values are slightly lower than the constraints of $\lambda\left(m_{t}\right) \lesssim 0.75$ and $\sqrt{\lambda^{2}\left(m_{t}\right)+\kappa^{2}} \lessgtr 0.75$ for the NMSSM that are often used as approximations to the RGE running [37]. ${ }^{13}$

\footnotetext{
${ }^{13}$ Note that these constraints can be weakened if one adds additional vectorlike matter, increasing the (negative) effect of the gauge couplings in (C3) [60-62,115].
}

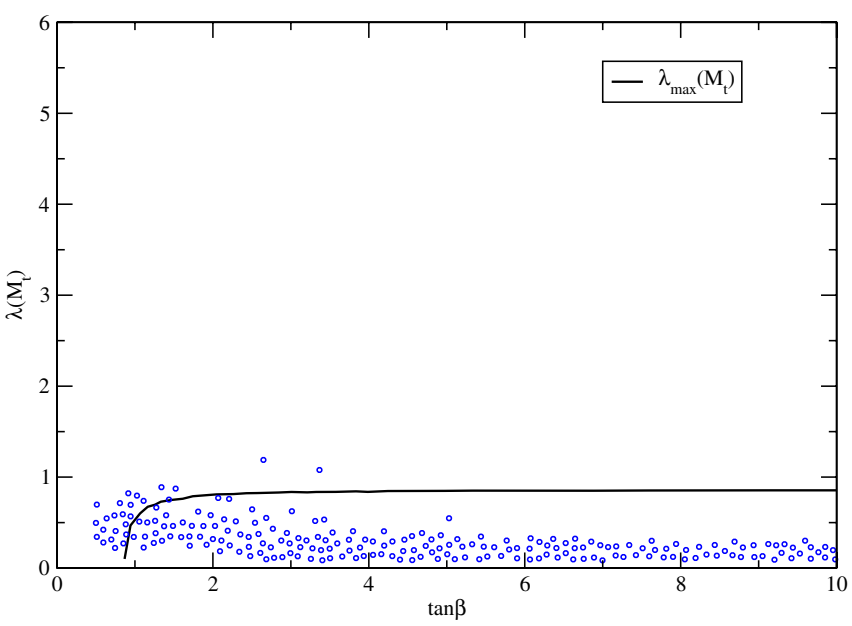

FIG. 11 (color online). Values of $\lambda$ vs $\tan \beta$ consistent with all considered constraints except perturbativity in scans over all parameters in the UMSSM, including $\theta_{E 6}$. The solid line represents the maximum allowed values of $\lambda\left(m_{t}\right)$ that remain perturbative for the particular choice of $\theta_{E 6}=\arctan \sqrt{15}$. Unlike the other models, $\lambda$ is strongly constrained by the requirement of a small $Z-Z^{\prime}$ mixing [37], which, for reasonable $\mu_{\text {eff }}$, favors large $\langle S\rangle$ and small $\lambda$. This is especially true for large $\tan \beta$ or small $\theta_{E_{6}}$, for which there are no cancellations between the contributions of $H_{d}^{0}$ and $H_{u}^{0}$ to the mixing.

The perturbativity limit for the UMSSM is given in Fig. 11 for the example ${ }^{14}$ of $\theta_{E 6}=\arctan \sqrt{15}$. It is less constraining than the NMSSM, with very few models being eliminated due to $\lambda_{\max }\left(m_{t}\right)$, which varies from $\sim 0.82$ to $\sim 0.86$, depending on the choice of $\theta_{E 6}$.

In addition to the perturbativity upper bound on $\lambda\left(m_{t}\right)$, the RGE running sets a lower limit of $\tan \beta \geq 1.8-1.9$ for the NMSSM/nMSSM and $\tan \beta \gtrsim 0.8-0.9$ for the UMSSM. Below these $\tan \beta$ values, a large $h_{t}$ often feeds into the running of $\lambda$, making $\lambda$ nonperturbative and sometimes resulting in a Landau pole below $M_{\mathrm{GUT}}[107,109-$ 111].

\footnotetext{
${ }^{14}$ The value for which the $U(1)^{\prime}$ charge of the right-handed neutrino $N_{i}^{c}$ vanishes.
}

[1] S. Dimopoulos and H. Georgi, Nucl. Phys. B193, 150 (1981).

[2] H. Baer and X. Tata, Weak Scale Supersymmetry: From Superfields to Scattering Events (University Press, Cambridge, England, 2004), p. 537
[3] M. Drees, R. Godbole, and P. Roy, Theory and Phenomenology of Sparticles: An account of fourdimensional $N=1$ Supersymmetry in High Energy Physics (World Scientific, Hackensack, USA, 2004), p. 555 . 
[4] N. Arkani-Hamed, S. Dimopoulos, and G. R. Dvali, Phys. Lett. B 429, 263 (1998).

[5] L. Randall and R. Sundrum, Phys. Rev. Lett. 83, 3370 (1999).

[6] N. Arkani-Hamed, A. G. Cohen, and H. Georgi, Phys. Lett. B 513, 232 (2001).

[7] N. Arkani-Hamed, A. G. Cohen, E. Katz, and A.E. Nelson, J. High Energy Phys. 07 (2002) 034.

[8] M. Schmaltz and D. Tucker-Smith, Annu. Rev. Nucl. Part. Sci. 55, 229 (2005).

[9] Z. Chacko, H.-S. Goh, and R. Harnik, Phys. Rev. Lett. 96, 231802 (2006).

[10] V. D. Barger, K. Whisnant, and W. Y. Keung, Phys. Rev. D 25, 291 (1982).

[11] H. Baer, A. Belyaev, T. Krupovnickas, and X. Tata, J. High Energy Phys. 02 (2004) 007.

[12] E. A. Baltz, M. Battaglia, M. E. Peskin, and T. Wizansky, Phys. Rev. D 74, 103521 (2006).

[13] D. Hooper and A. M. Taylor, J. Cosmol. Astropart. Phys. 03 (2007) 017.

[14] M. Carena, D. Hooper, and A. Vallinotto, Phys. Rev. D 75, 055010 (2007).

[15] J. L. Feng, S. Su, and F. Takayama, Phys. Rev. Lett. 96, 151802 (2006).

[16] R. Arnowitt et al., arXiv:hep-ph/0701053.

[17] A. Birkedal, K. Matchev, and M. Perelstein, Phys. Rev. D 70, 077701 (2004).

[18] V. Cirigliano, S. Profumo, and M. J. Ramsey-Musolf, J. High Energy Phys. 07 (2006) 002.

[19] H. Baer et al. (unpublished).

[20] J. D. Vergados, C. C. Moustakidis, and V. Oikonomou, AIP Conf. Proc. 878, 138 (2006).

[21] L. Bergstrom, J. Edsjo, and P. Ullio, Phys. Rev. Lett. 87, 251301 (2001).

[22] L. Bergstrom, P. Ullio, and J. H. Buckley, Astropart. Phys. 9, 137 (1998)

[23] V. D. Barger, F. Halzen, D. Hooper, and C. Kao, Phys. Rev. D 65, 075022 (2002).

[24] M. Cirelli et al., Nucl. Phys. B727, 99 (2005).

[25] S. Profumo and A. Provenza, J. Cosmol. Astropart. Phys. 12 (2006) 019.

[26] J. Silk and M. Srednicki, Phys. Rev. Lett. 53, 624 (1984).

[27] E. A. Baltz, J. Edsjo, K. Freese, and P. Gondolo, Phys. Rev. D 65, 063511 (2002).

[28] C. J. Hailey et al., J. Cosmol. Astropart. Phys. 01 (2006) 007.

[29] K. Mori et al., Astrophys. J. 566, 604 (2002).

[30] P. Langacker and M.-x. Luo, Phys. Rev. D 44, 817 (1991).

[31] J. R. Ellis, S. Kelley, and D. V. Nanopoulos, Phys. Lett. B 260, 131 (1991).

[32] U. Amaldi, W. de Boer, and H. Furstenau, Phys. Lett. B 260, 447 (1991).

[33] J. E. Kim and H. P. Nilles, Phys. Lett. 138B, 150 (1984).

[34] E. Accomando et al., arXiv:hep-ph/0608079.

[35] T. Han, P. Langacker, and B. McElrath, Phys. Rev. D 70, 115006 (2004).

[36] V. Barger, P. Langacker, and G. Shaughnessy, Phys. Lett. B 644, 361 (2007)

[37] V. Barger, P. Langacker, H.-S. Lee, and G. Shaughnessy, Phys. Rev. D 73, 115010 (2006).
[38] V. Barger, P. Langacker, and H.-S. Lee, Phys. Lett. B 630, 85 (2005).

[39] V. Barger, P. Langacker, and G. Shaughnessy, Phys. Rev. D 75, 055013 (2007).

[40] V. Barger, P. Langacker, and G. Shaughnessy, arXiv:hep$\mathrm{ph} / 0702001$.

[41] S. Y. Choi, D. J. Miller, and P. M. Zerwas, Nucl. Phys. B711, 83 (2005).

[42] S. Y. Choi, H. E. Haber, J. Kalinowski, and P. M. Zerwas, arXiv:hep-ph/0612218.

[43] J. F. Gunion, D. Hooper, and B. McElrath, Phys. Rev. D 73, 015011 (2006).

[44] U. Ellwanger, J. F. Gunion, C. Hugonie, and S. Moretti, arXiv:hep-ph/0401228.

[45] A. Arhrib, K. Cheung, T.-J. Hou, and K.-W. Song, J. High Energy Phys. 03 (2007) 073.

[46] P. Fayet, Nucl. Phys. B90, 104 (1975).

[47] P. Fayet, Phys. Lett. 69B, 489 (1977).

[48] J. R. Ellis, J. F. Gunion, H. E. Haber, L. Roszkowski, and F. Zwirner, Phys. Rev. D 39, 844 (1989).

[49] M. Bastero-Gil, C. Hugonie, S. F. King, D. P. Roy, and S. Vempati, Phys. Lett. B 489, 359 (2000).

[50] A. de Gouvea, A. Friedland, and H. Murayama, Phys. Rev. D 57, 5676 (1998).

[51] C. Panagiotakopoulos and K. Tamvakis, Phys. Lett. B 469, 145 (1999).

[52] C. Panagiotakopoulos and A. Pilaftsis, Phys. Rev. D 63, 055003 (2001).

[53] A. Menon, D.E. Morrissey, and C.E. M. Wagner, Phys. Rev. D 70, 035005 (2004).

[54] A. Dedes, C. Hugonie, S. Moretti, and K. Tamvakis, Phys. Rev. D 63, 055009 (2001).

[55] M. Cvetic, D. A. Demir, J. R. Espinosa, L. L. Everett, and P. Langacker, Phys. Rev. D 56, 2861 (1997).

[56] P. Langacker and J. Wang, Phys. Rev. D 58, 115010 (1998).

[57] B. de Carlos and J. R. Espinosa, Phys. Lett. B 407, 12 (1997).

[58] J. Erler, P. Langacker, and T.-j. Li, Phys. Rev. D 66, 015002 (2002).

[59] T. Li, arXiv:hep-ph/0612359.

[60] S. F. King, S. Moretti, and R. Nevzorov, Phys. Rev. D 73, 035009 (2006).

[61] S. F. King, S. Moretti, and R. Nevzorov, Phys. Lett. B 634, 278 (2006).

[62] S.F. King, S. Moretti, and R. Nevzorov, arXiv:hep-ph/ 0701064.

[63] R. Blumenhagen, B. Kors, D. Lust, and S. Stieberger, arXiv:hep-th/0610327.

[64] R. Blumenhagen, S. Moster, and T. Weigand, Nucl. Phys. B751, 186 (2006).

[65] R. Blumenhagen, M. Cvetic, P. Langacker, and G. Shiu, Annu. Rev. Nucl. Part. Sci. 55, 71 (2005).

[66] A. E. Faraggi, E. Manno, and C. Timirgaziu, arXiv:hep-th/ 0610118.

[67] D. O'Connell, M.J. Ramsey-Musolf, and M. B. Wise, Phys. Rev. D 75, 037701 (2007).

[68] O. Bahat-Treidel, Y. Grossman, and Y. Rozen, arXiv:hep$\mathrm{ph} / 0611162$.

[69] J. McDonald, Phys. Rev. D 50, 3637 (1994).

[70] C. P. Burgess, M. Pospelov, and T. ter Veldhuis, Nucl. 
Phys. B619, 709 (2001).

[71] X.-G. He, T. Li, X.-Q. Li, and H.-C. Tsai, arXiv:hep-ph/ 0701156.

[72] F. Ferrer, L. M. Krauss, and S. Profumo, Phys. Rev. D 74, 115007 (2006).

[73] D. G. Cerdeno, C. Hugonie, D. E. Lopez-Fogliani, C. Munoz, and A. M. Teixeira, J. High Energy Phys. 12 (2004) 048.

[74] D. G. Cerdeno, E. Gabrielli, D.E. Lopez-Fogliani, C. Munoz, and A. M. Teixeira, arXiv:hep-ph/0701271.

[75] W. M. Yao et al. (Particle Data Group), J. Phys. G 33, 1 (2006).

[76] D. N. Spergel et al., arXiv:astro-ph/0603449.

[77] V. Barger, M. S. Berger, and P. Ohmann, Phys. Rev. D 47, 1093 (1993).

[78] J. R. Ellis, K. A. Olive, Y. Santoso, and V. C. Spanos, Phys. Rev. D 71, 095007 (2005).

[79] H. Baer, C. Balazs, A. Belyaev, and J. O'Farrill, J. Cosmol. Astropart. Phys. 09 (2003) 007.

[80] R. Trotta, R. R. de Austri, and L. Roszkowski, New Astron. Rev. 51, 316 (2007).

[81] J. R. Ellis, D. Nanopoulos, K. A. Olive, and Y. Santoso, Phys. Lett. B 633, 583 (2006).

[82] R. Arnowitt, B. Dutta, T. Kamon, N. Kolev, and D. Toback, Phys. Lett. B 639, 46 (2006).

[83] V. Barger, C. Kao, P. Langacker, and H.-S. Lee, Phys. Lett. B 600, 104 (2004).

[84] P. Gondolo et al., J. Cosmol. Astropart. Phys. 07 (2004) 008.

[85] G. Belanger, F. Boudjema, A. Pukhov, and A. Semenov, Comput. Phys. Commun. 176, 367 (2007).

[86] ISATOOLS can be found in the unpublished manual on the following web site: http://www.hep.fsu.edu/ isajet/ isajet775.ps.

[87] G. Jungman, M. Kamionkowski, and K. Griest, Phys. Rep. 267, 195 (1996).

[88] J. Edsjo and P. Gondolo, Phys. Rev. D 56, 1879 (1997).

[89] B. McElrath, Phys. Rev. D 72, 103508 (2005).

[90] A. B. Lahanas and V. C. Spanos, Eur. Phys. J. C 23, 185 (2002).

[91] J. R. Ellis, T. Falk, G. Ganis, K. A. Olive, and M.
Srednicki, Phys. Lett. B 510, 236 (2001).

[92] J.L. Feng, S.-f. Su, and F. Takayama, arXiv:hep-ph/ 0410119.

[93] J.L. Feng, K. T. Matchev, and F. Wilczek, Phys. Lett. B 482, 388 (2000).

[94] H. Baer, T. Krupovnickas, S. Profumo, and P. Ullio, J. High Energy Phys. 10 (2005) 020.

[95] D.S. Akerib et al. (CDMS), Phys. Rev. D 73, 011102 (2006).

[96] W. J. Bolte et al., J. Phys.: Conf. Ser. 39, 126 (2006).

[97] V. Sanglard et al. (EDELWEISS), Phys. Rev. D 71, 122002 (2005).

[98] D. S. Akerib et al., Nucl. Instrum. Methods Phys. Res., Sect. A 559, 390 (2006).

[99] P. Benetti et al., arXiv:astro-ph/0701286.

[100] J. R. Ellis, A. Ferstl, and K. A. Olive, Phys. Lett. B 481, 304 (2000).

[101] P. Sikivie, Phys. Lett. B 567, 1 (2003).

[102] M.E. Gomez, T. Ibrahim, P. Nath, and S. Skadhauge, Phys. Rev. D 74, 015015 (2006).

[103] K. Hagiwara, A. D. Martin, D. Nomura, and T. Teubner, arXiv:hep-ph/0611102.

[104] M. Davier, arXiv:hep-ph/0701163.

[105] V. Barger, C. Kao, P. Langacker, and H. Lee, Phys. Lett. B 614, 67 (2005).

[106] P. Langacker and J. Wang, Phys. Rev. D 58, 115010 (1998).

[107] G. K. Yeghian, arXiv:hep-ph/9904488.

[108] S. P. Martin and M. T. Vaughn, Phys. Rev. D 50, 2282 (1994).

[109] U. Ellwanger and C. Hugonie, Eur. Phys. J. C 25, 297 (2002).

[110] U. Ellwanger and C. Hugonie, arXiv:hep-ph/0006222.

[111] T. Elliott, S. F. King, and P. L. White, Phys. Rev. D 49, 2435 (1994).

[112] P. Binetruy and C.A. Savoy, Phys. Lett. B 277, 453 (1992).

[113] K. S. Babu, C. F. Kolda, and J. March-Russell, Phys. Rev. D 54, 4635 (1996).

[114] S. Bethke, Prog. Part. Nucl. Phys. 58, 351 (2007).

[115] J. Kang and P. Langacker (unpublished). 